\title{
Preferences for the Resolution of Uncertainty and the Timing of Information
}

\author{
Kirby Nielsen*
}

Draft: April 2, 2018

\begin{abstract}
We present results from a laboratory experiment designed to identify preferences over the resolution of uncertainty and timing of non-instrumental information acquisition in a rich choice set. We test theoretical predictions along three dimensions: early vs. late resolution, one-shot vs. gradual, and positive vs. negative skew. Treatments very whether the outcome previously has been determined, as in an information structure, or is determined later, as in a compound lottery. We find that individuals prefer to delay uncertainty resolution when the outcome has not been determined and prefer to expedite uncertainty resolution when the outcome has already occurred. We find no evidence for a preference for one-shot resolution in either context.
\end{abstract}

KEYWORDS: Resolution of uncertainty; Information preferences; Anticipatory emotions

\section{INTRODUCTION}

People naturally face uncertainty in almost every aspect of daily life. In the presence of the unknown, individuals may seek out information to reduce their uncertainty or resolve it earlier. Often, reducing uncertainty is utilitarian-in expectation, better information leads to better decisions. In this vein, economists typically value information only insofar as it provides instrumental value to decision makers. Standard theory predicts that individuals are only willing to pay for information that can inform subsequent decisions and also predicts that individuals will never have an incentive to avoid information 1

However, recent experimental evidence suggests that individuals have strict preferences over noninstrumental information, or information that has no value in decision making. To understand the domain of these preferences, imagine a genetic test that can reveal to you whether you're at a high or low risk of developing a disease. However, the test may not be conclusive-ultimately, you might only know for sure when you do (or don't) develop the condition later in life. Would you want to know the test results? Or would you

\footnotetext{
*Department of Economics, The Ohio State University, 410 Arps Hall, 1945 N. High Street, Columbus, Ohio 43210, USA. For helpful conversations and comments, I thank Yaron Azrieli, Puja Bhattacharya, Alex Brown, Evan Calford, Katie Coffman, Ritesh Jain, John Kagel, Ian Krajbich, Matthew McMahon, and Collin Raymond. I am especially beholden to Paul J. Healy for his time, advice, and encouragement. I also thank participants at the 2018 Spring School in Behavioral Economics, Summer School of the Econometric Society 2017, Midwest Economic Association 2017 meeting, the Economic Science Association 2017 North America Meeting, and the Ohio State Theory Colloquium for many helpful comments. Research support was provided by the Decision Science Collaborative and Journal of Money, Credit, and Banking Small Research Grants, Ohio State University.

${ }^{1}$ This does not take into account environments where information avoidance provides strategic benefits. See Poulsen and Roos 2010 , for an example of experimental evidence on strategic information avoidance.
} 
rather just wait to find out whether you develop the condition?

Of course there are instrumental considerations at play. Knowing this information could affect lifestyle choices, savings decisions, insurance coverage, etc. But it's not hard to imagine non-instrumental considerations playing a role in the decision as well. Even if knowing the test results wouldn't affect any subsequent choices, it's likely that individuals are not indifferent to this type of information. Taking the test might induce a great deal of anxiety, disappointment, or hope, among other emotional responses, which can enter directly into interim utility. As a result, individuals may have preferences over information itself, absent the role information plays in decision making.

Recent theoretical literature has considered modeling and axiomatizing preferences over uncertainty resolution and non-instrumental information. Various models have incorporated behavioral phenomena to predict preferences along three dimensions: i) one-shot vs. gradual resolution, ii) early vs. late resolution, iii) and positive vs. negative skewness. An individual with a preference for gradual resolution of uncertainty would prefer to receive information in small pieces over time. On the other hand, an individual with a preference for one-shot resolution dislikes gradual information and would rather all uncertainty resolve at once, regardless of when that is. A preference for early resolution corresponds, naturally, to a desire for information as soon as possible, and a preference for late resolution indicates a desire to avoid information. Positively skewed information has the potential to eliminate more uncertainty about the desired outcome while negatively skewed information eliminates more uncertainty about the undesired outcome.

To put these preferences in the context of our health test example, an individual with a preference for oneshot resolution would only take a test that were perfectly informative or perfectly uninformative. Otherwise, he would avoid the test, preferring to wait and learn the disease outcome for sure at a time when all uncertainty could be resolved at once. Theoretically, these preferences could stem from disappointment aversion (Palacios-Huerta, 1999), loss aversion over changes in beliefs (Koszegi and Rabin, 2009), or the certainty effect (Dillenberger, 2010). An individual preferring gradual resolution would choose a test that eliminates some, but not all, uncertainty about the disease. A preference for early resolution corresponds to a desire to take the most accurate test, eliminating as much uncertainty as possible, while a preference for late resolution leads to choosing a less accurate test. An individual preferring positive skew would choose a test that often gives false positives, but he can be very confident he will not develop the condition if the test result is negative. That is, he prefers eliminating uncertainty about the good outcome. A preference for negative skew is just the opposite - this individual would choose a test where a negative test result is relatively uninformative, but he can be very sure he will develop the condition if the test is positive. He prefers eliminating uncertainty about the bad outcome.

To date, there is limited clean experimental evidence on preferences over uncertainty resolution, partic- 
ularly preferences for one-shot vs. gradual information. Experimental results are sparse and often appear contradictory, so there is no empirical consensus on how to characterize preferences over information and uncertainty resolution. One factor leading to this lack of consensus is that uncertainty resolution is studied in two different, though theoretically equivalent, domains-multi-stage lotteries (Boiney, 1993, Eliaz and Schotter, 2010, Aria, 1997; Ahlbrecht and Weber, 1997; Lovallo and Kahneman, 2000; Von Gaudecker et al., 2011; Brown and Kim, 2013, Kocher et al., 2014; Zimmermann, 2015) and information structures (Chew and Ho, 1994: Ganguly and Tasoff, 2016, Falk and Zimmermann, 2016; Masatlioglu et al., 2017). While a one-to-one mapping exists between the two environments, a key difference lies in when the outcome in question occurs. In a multi-stage lottery, uncertainty resolves throughout with the outcome ultimately determined in the last stage. This is more akin to a test which could reveal the presence of a gene which puts you at greater risk for developing a condition, such as Alzheimer's, in the future $2^{2}$ On the other hand, an information structure generates signals about an outcome that has already been determined. This is closer to a test which detects the presence of a disease, like HIV, which the individual may possess already. While theoretically identical, individuals may perceive uncertainty that has been resolved differently than uncertainty which has not yet determined ${ }^{3}$ Our experiment allows us to compare these two domains to address some of the discrepancies and fill recent gaps in the literature. Additionally, identifying differences in preferences over uncertainty in these two environments can help us identify in which field settings information avoidance will be most likely, and therefore where to focus greater external incentives for information acquisition.

To identify these preferences experimentally, we allow subjects to choose their most preferred two-stage lottery or information structure that has a fixed prior $50 \%$ chance of giving a high prize and $50 \%$ chance of giving a low prize. In the Lottery treatment, we first choose one of two "urns," each of which contains some red and blue balls. After a time delay, we draw a ball from the chosen urn. The color of the ball determines the payoff-relevant outcome for the subject. The experiment gives subjects the opportunity to choose how much uncertainty to resolve in the first and second stages by choosing different urn compositions. In the Information Structure treatment, we first show a red or blue ball as a signal, and after a time delay reveal the urn from which it was drawn, corresponding to the high or low prize. Subjects choose their most preferred two-stage lottery or information structure from the set of all lotteries or information structures that have the same probability over final outcomes (e.g. probability of winning the high or low prize) and differ only in the timing

\footnotetext{
${ }^{2}$ Direct-to-consumer genetic testing services are quickly gaining popularity. For example, 23andMe gives information on ancestry and certain wellness traits. In addition, the service provides tests for genetic variants which correlate with increased risk of developing ten different conditions including Parkinsons, Alzheimer's, and celiac. Test results indicate number of variants detected and the resulting posterior likelihood of developing the disease, similar to the two-stage uncertainty resolution in our design. Consumers now are required specifically to opt into these tests, since many individuals may prefer not to know this information. In fact, a survey of individuals who took the test before it required an opt in found that $13 \%$ of individuals given the information on Alzheimer's chose not to look at it Burling 2017).

${ }^{3}$ In particular, Rothbart and Snyder 1970 ) find differences in risk taking across these two domains. The authors suggest that "illusion of control" over future outcomes increases perceived likelihood of favorable events occurring.
} 
of uncertainty resolution. We place additional restrictions on the set of possible choices in order to disentangle different types of information preferences.

We find that preferences over lotteries differ significantly from preferences over equivalent information structures. In both treatments, we find intermediate resolution preferred to both the earliest and latest resolution. Among those who do not choose intermediate resolution, in the Information treatment we see a strong and persistent preference for early resolution. Regardless of whether early resolution is gradual or one-shot, and regardless of skew, choosing the most informative signal structure is by far the modal choice. For lotteries, we find more heterogeneous preferences. Choices never exhibit a significant preference for early resolution, and results often favor late resolution and information avoidance. We find little evidence for a preference for one-shot resolution in either treatment. Finally, we replicate and extend existing results in the literature on skewness. Individuals prefer positively skewed information in a more restricted choice set (in line with results from Masatlioglu et al., 2017), though this does not seem to generalize to completely unrestricted choices. Using willingness to pay elicitation, we conclude that the results above reflect strict preferences and are not driven by indifference. More than $70 \%$ of subjects are willing to pay for their preferred manner of uncertainty resolution despite understanding that their choice cannot affect uncertain outcomes.

Our design features several important characteristics. First, we run two analogous treatments to compare directly preferences over lotteries to those over information structures. The only meaningful difference between the two treatments is that the outcome is determined at the end of the two-stage lottery while it happens at the beginning to generate signals in the information treatment. Second, all information is noninstrumental and cannot influence or inform any subsequent decisions. We carefully set up the experimental environment to ensure that information cannot confer any strategic, ego-relevant, or otherwise tangible advantages. As a result, observed preferences reflect true preferences over uncertainty resolution and are not confounded by other concerns. Third, we include a controlled but meaningful time delay between stages of uncertainty resolution in order to represent real resolution over time. Finally, we move beyond binary choices, which have been the focus of the literature to-date. Individuals express preferences over an entire space of actuarially-equivalent lotteries or information structures, capturing the whole range of intermediate uncertainty resolution. This allows us to test theories rigorously, as they predict preferences over all possible manners of uncertainty resolution. Thus, we can attribute subjects' choices as a true maximum of preference, rather than inferring direction of preference from a set of binary choices.

Our results contribute to the literature in several ways. Ours is the first experiment to compare information structures to analogous compound lotteries. The previous literature has studied both independently, failing to come to consensus on preferences over the resolution of uncertainty. Some papers have found a preference for early resolution (Eliaz and Schotter, 2007; Falk and Zimmermann, 2016, Masatlioglu et al., 2017), some late 
(Ganguly and Tasoff, 2016, Kocher et al., 2014), and some find more mixed evidence (Zimmermann, 2015). We conclude that preferences look very different in lotteries, when the outcome is determined at the end, compared to information structures, when the outcome has already been determined. Our results suggest that the previous literature appears inconsistent because these papers have studied both lotteries and information structures without distinguishing between the two. Both environments warrant attention and more focused study, but researchers should consider which framework better suits their motivation when designing experiments and applying results to external environments. Information avoidance has long been a puzzling concern for economists, and our results suggest that information avoidance will be most common over outcomes to be resolved in the future. This insight can help design and present efficient information to individuals in a way most likely to lead to informed decisions.

Finally, it's worth taking a moment to discuss the relevance of studying preferences over uncertainty resolution and non-instrumental information. Precisely because the information is non-instrumental, these preferences in isolation have no economic consequences by any standard definition. Individuals have no decisions to make, so their choice of uncertainty resolution cannot affect outcomes. However, we should not, then, conclude that preferences over non-instrumental information are irrelevant to economic analysis. First, there are many factors in the world that interact with the simple decision to acquire or avoid information, whether it be instrumental or not. For example, medical tests are costly, so refusing to get tested could be influenced by budget in addition to, or instead of, not wanting to know. Because of these external factors, we cannot determine individuals' pure preferences over information just by looking at observable decisions. So while we observe many instances of "information avoidance" (see Golman et al., 2016 for a recent survey), it may be for reasons other than preferences over information. Therefore, clean experimental investigation of non-instrumental information is necessary for insight into preferences over uncertainty, absent these other considerations.

Perhaps more fundamentally, information we wish to study is often instrumental; we generally assume acquiring gradual information will result in better interim decision making. An underlying aversion to a particular type of information may therefore lead to information avoidance at the expense of informed decision making: ${ }^{4}$ It is important to understand these underlying behavioral preferences over information in order to analyze these environments and present information to decision makers in a utility-maximizing way. In short, non-instrumental preferences can affect instrumental decision making, so we should understand these preferences to build better descriptive models of decision making under uncertainty.

\footnotetext{
${ }^{4}$ This does not follow directly. One could imagine an individual who avoids information about events where he can't take any action, but would prefer to know if given the chance to do something about it.
} 


\section{LiteratuRE}

In this section, we outline the theoretical literature and papers predicting preferences along one or more dimension of uncertainty resolution. We follow with a summary of the experimental literature testing the predictions of these theories.

\section{Theoretical Predictions}

As described above, there are three interesting dimensions of information preference: early vs. late, one-shot vs. gradual, and positive vs. negative skewness. Of course, standard theory predicts that individuals are indifferent along all three dimensions-an expected utility maximizer satisfies Reduction of Compound Lotteries, leading to indifference over all actuarially-equivalent lotteries. However, recent theories have relaxed this assumption, and below we outline these models and the choices they prescribe in our domain.

EARLY VS. LATE. - Most of the early theoretical work on preferences over non-instrumental information focused on the preference for or against receiving earlier, or more informative, non-instrumental information. Early models such as Kreps and Porteus (1978), Epstein and Zin (1989), and Grant et al. (1998) categorize utility that leads to preference for early or late resolution. These models characterize conditions on an individual's local utility function leading to a preference for early or late resolution. As such, they can accommodate preference for early or late resolution, though most experimental evidence shows a preference for early resolution, as outlined below.

Loewenstein (1987) models a decision maker who derives direct utility from anticipation. He shows that these anticipatory emotions lead to a preference for postponing pleasure and expediting pain. This decision maker would prefer to experience losses early on to "get them over with," while deriving positive anticipatory utility from looking forward to delayed gains in the future. In contrast to the models above, this would predict a preference for late resolution of uncertainty about the outcome of a lottery with positive payoffs, like the lottery in our experiment.

ONE-SHOT VS. GRADUAL. - Palacios-Huerta (1999) was one of the first to study preferences over one-shot versus gradual resolution of uncertainty. Building on the disappointment aversion model of Gul (1991), PalaciosHuerta (1999) shows how a disappointment averse decision maker is averse to the sequential resolution of uncertainty. Gradual resolution of uncertainty exposes the decision maker to more opportunities at which he could experience disappointment, and therefore he prefers all uncertainty resolve at once.

Following closely-related intuition, Köszegi and Rabin (2009) introduce a theory of reference-dependent 
preferences which makes similar predictions in the context of uncertainty resolution. Köszegi and Rabin's decision maker is loss averse over changes in beliefs. The process of gradual resolution of uncertainty exposes the decision maker to greater opportunity for belief fluctuations. Since the decision maker is loss averse over these belief fluctuations, downward belief adjustments bring a greater utility loss than the corresponding gain of upward adjustments. As a result, the theory similarly predicts individuals will dislike gradual resolution and instead prefer one-shot resolution of uncertainty. Kőszegi and Rabin's model also incorporates anticipatory utility over time, assuming that decision makers prefer to receive disappointing news further from the time it will be realized. This gives a weak prediction that individuals would prefer to resolve uncertainty all at once as soon as possible, but will prefer any one-shot resolution over gradual.

Most recently, Dillenberger (2010) formally models uncertainty resolution using two-stage lotteries. In his model, he assumes the decision maker satisfies Time Neutrality (Segal, 1990) and therefore is indifferent between all uncertainty resolving in the first stage and all uncertainty resolving in the second stage, but prefers both of those to any gradually resolving lottery with the same prior. Dillenberger's predictions stem from his foundational axiom, Negative Certainty Independence (NCI). NCI formalizes the "certainty effect," where individuals have a disproportionate preference for certain over uncertain outcomes (Allais, 1953, Kahneman and Tversky, 1979). This certainty preference drives the distaste for gradual resolution in Dillenberger's framework. As a result, Dillenberger (2010) predicts that individuals satisfying NCI will always choose to resolve all uncertainty in a single stage of the lottery.

While most models specifically addressing this dimension predict a preference for one-shot over gradual resolution, Ely et al. (2015) develop a theoretical model of preference for suspense and surprise which predicts the opposite. According to their model, uncertainty resolution leads to higher suspense if the variance in beliefs is larger, and leads to higher surprise if the gap between one period's beliefs to the next period's beliefs is larger. They assume individuals have a direct preference for suspense and/or surprise, and their results predict that one-shot resolution can be least preferred.

SKEWnESS. - Masatlioglu et al. (2017) discuss the predictions that these various theories make regarding preferences over skewness of information. In particular, the theories of Köszegi and Rabin (2009) and Ely et al. (2015) both require individuals to be indifferent to skewness, while Grant et al. (1998) and Gul (1991) can accommodate strict preference in either direction. We direct the interested reader to Masatlioglu et al. (2017) for details.

Table 1 summarizes the theoretical predictions in these three dimensions. 


\begin{tabular}{lccc}
\hline & Early v. Late & One-Shot v. Gradual & Positive v. Negative Skew \\
\hline Segal 1990 & Indifferent & Indifferent & Indifferent \\
Kreps Porteus 1978 & Earliest or Latest & & Either \\
Grant et al. 1998 & Either & & \\
Köszegi Rabin 2009 & Early & One-Shot & Indifferent \\
Dillenberger 2010 & & One-Shot & \\
Ely et al. 2013 & Local Late & Gradual & Indifferent \\
\hline
\end{tabular}

Table I: Summary of Theoretical Predictions

\section{Experimental Evidence}

The experimental literature on preferences over non-instrumental information and resolution of uncertainty began with focus on the distinction between early and late resolution. Most experimental papers directly test preferences over lotteries and find that individuals typically prefer early resolution of uncertainty (Boiney, 1993: Chew and Ho, 1994; Ahlbrecht and Weber, 1997; Aria, 1997; Lovallo and Kahneman, 2000). However, these studies use hypothetical stakes and do not control for the fact that early resolution could have instrumental value. More recent papers (Von Gaudecker et al., 2011; Brown and Kim, 2013; Kocher et al., 2014; Meissner, 2017) incentivize choices but still implement significant uncontrolled time delays such that information could be considered instrumental. These papers generally find a stronger preference for early over late resolution, but this could be confounded with planning benefits of information.

There are few papers studying truly non-instrumental information in incentivized experiments. Ganguly and Tasoff (2016) elicit willingness to pay to expedite or delay learning the outcome of a pre-determined lottery. They find that preferences depend on the stakes of the lottery ${ }^{5}$ With low stakes, individuals prefer to delay resolution. Preference for early resolution becomes stronger as the potential prize of the lottery increases. Ganguly and Tasoff also look at preferences to acquire or avoid information about the outcome of HIV tests and find that individuals are more likely to avoid getting tested for the more serious strand of the virus compared to the treatable one ${ }^{6}$

A recent paper by Falk and Zimmermann(2016) also studies preferences over early versus late resolution in the "loss" domain, taking away all instrumental value of information. Subjects were randomized into receiving electric shocks or not and could choose when to learn whether they would be shocked. Their fate had already been determined, so the only choices was in waiting to learn the information or learning it immediately. They find a stronger preference for early resolution of uncertainty, just as we see in the information structure treatment. Their paper also studies the effect of attention. When subjects are given a distraction task to

\footnotetext{
${ }^{5}$ While this choice is framed as a "lottery," the lottery has already played out. Under the language used in this paper, this this would be more consistent with an information structure.

${ }^{6}$ They do not control for possible instrumental value of these test results.
} 
occupy their attention during the waiting period, they preferred not to know whether they would receive shocks. However, when this distraction was not available, they preferred to know earlier whether they would receive shocks.

As the focus in the theory literature shifted to studying preferences over one-shot versus gradual resolution, a recent experimental literature has emerged alongside. The experimental evidence in this domain is sparse and inconclusive. Zimmermann (2015) directly tests the theory of Köszegi and Rabin (2009) by asking whether individuals prefer to get information "clumped" or "piecewise." In his experiment, subjects are entered into a lottery whose outcome will be determined by the rolls of 3 dice. Subjects were given the option of learning all 3 die rolls on the first day, learning one die roll each day, or learning all 3 on the last day 7 He finds heterogeneous preferences, with no clear preference for early clumped information as Köszegi and Rabin(2009) predict, consistent with our results in the compound lottery treatment. A sizable fraction of subjects do prefer gradual and late information, in contrast to the early evidence finding preference for early resolution.

Eliaz and Schotter (2007, 2010) analyze an environment where subjects have to make a guess about the state of the world and are given the opportunity to purchase information before guessing. However, the experiment is set up such that the information will not influence subjects' decisions; thus, it is entirely noninstrumental. Despite this, they find a large proportion of participants are willing to purchase the information, even though subjects understand the lack of instrumental value. The authors suggest that individuals are willing to purchase information that makes them more confident in their decisions. In purchasing information, however, individuals are choosing to expose themselves to gradual resolution without gaining any instrumental value. This paper does not introduce any substantial time delay between the stages of resolution, so the environment is quite different from that of the current study.

The closest paper to ours and most recent experimental evidence is Masatlioglu et al. (2017). Masatlioglu et al. broadly investigate preferences over information structures using binary choices. Their primary interest is in skewness, focusing on whether individuals prefer positively- or negatively-skewed information about the outcome of a lottery. Their main conclusion is that individuals prefer early information and this preference supersedes preference for skewness or aversion to gradual information. When information structures are equally informative, individuals exhibit a preference for positively skewed information.

Our paper contributes to the experimental literature in a number of ways. First, previous studies differ in whether they focus on compound lotteries or information structures. Though theoretically equivalent, empirical equivalence on lotteries and information structures has not been established. We find the two environments are not equivalent. Second, many of the previous tests of uncertainty preference conflate preferences

\footnotetext{
${ }^{7}$ Note, this allows for information to have instrumental value. Since individuals receive payment from the lottery days in the future, they might prefer to know how much they will receive so they can delay spending until after receiving the money, decline an extra shift at work knowing they will soon receive a large windfall, etc.
} 
for one-shot resolution with preference for early or late information. We design our experiment specifically to disentangle these preferences and separate preference for one-shot vs. gradual from preferences for early vs. late resolution, as we explain in Section IV] Third, we employ a "budget set" design rather than eliciting binary choices, which allows us to gather more information on preferences. Theories that predict preferences for one-shot resolution, for example, predict that individuals will prefer this to any other form of resolution. Our design allows subjects to choose their most preferred resolution from the entire space of possibilities, rather than relying on binary choices to infer preferences.

\section{FRAMEWORK}

In this section, we outline the theoretical framework and lottery spaces we investigate. We derive testable implications of theoretical predictions in this domain, to be analyzed in Section $\mathrm{V}$, The experimental design will follow closely the theoretical framework.

\section{Preliminaries}

To simplify exposition and maintain parity with our experimental design, we assume there are two states of the world corresponding to two prizes - a high prize $(\mathrm{H})$ and a low prize $(\mathrm{L})$. There are three relevant time periods, $t$, in the model: $\{0,1,2\}$. Preferences are defined over the space of two-stage lotteries or information structures with objective probabilities. At time 0 , all individuals have the same prior, $\operatorname{pr}(H)=\theta$. Some uncertainty may resolve at $t=1$, resulting in a new posterior at time 2 . All uncertainty resolves by the end of period $t=2$, leaving no uncertainty about the state. This describes a general framework of two-stage lotteries or information structures, and we present the detailed theoretical framework in the language of our experiment.

\section{Two-Stage Lotteries}

We conceptualize the lottery as drawing a colored ball from an urn. At time $t=1$, the lottery selects either Urn 1 or Urn 2, according to probabilities $(p, 1-p)$. Each urn can contain some fraction of red and blue balls. At time $t=2$, the lottery selects a ball from the urn, where selecting a red ball corresponds to winning the high prize and selecting a blue ball corresponds to winning the low prize. From Urn 1, a red ball is drawn with probability $q$ and a blue ball with probability $1-q$. From Urn 2 , a red ball is drawn with probability $r$ and a blue ball with probably $1-r 8$ While a time delay is not explicitly modeled, we use this framework to describe situations where meaningful time can pass in between Stage 1 and Stage 2 realizations, so the

\footnotetext{
${ }^{8}$ Naturally, we require $p \in[0,1], q \in[0,1]$, and $r \in[0,1]$.
} 
duration between $t=1$ and $t=2$ is substantial. This compound lottery framework is shown in Figure $\mathrm{I}$

All $(p, q, r)$ such that $p q+(1-p) r=\theta$ give the same ex-ante likelihood of winning the high and low prizes; the difference lies in how uncertainty is resolved in Stage 1 relative to Stage 2. Without loss of generality, we focus on situations where $q \leq \theta$ and $r \geq \theta{ }^{9}$ As a result, seeing Urn 1 in Stage 1 of the lottery represents bad news and seeing Urn 2 represents good news-with probability $p$, the posterior is reduced from $\theta$ to $q$ after Stage 1 and with probability $1-p$ the posterior is increased from $\theta$ to $r$. Uncertainty resolution is completely characterized by $(p, q, r)$, which will be the choice variables in our experiment.

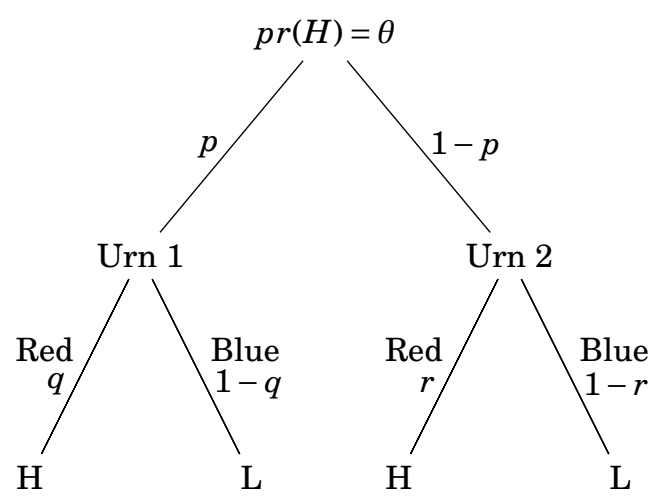

Figure I: Two-stage Lottery

Graphically, we depict the space of all such lotteries in Figure II shown with $\theta=0.5$, which is the prior used in our experimental design. The y-axis plots $q$, the probability of drawing the high prize from Urn 1 , and the x-axis plots $r$, the probability of drawing the high prize from Urn 2 . The gradient plots $p$, the probability of selecting Urn 1 in the first stage. Any point in this $(p, q, r)$ space represents a two-stage lottery with $\theta=0.5$.

ONE-SHOT VS. GRADUAL RESOLUTION. - In this framework, one-shot resolution of uncertainty precisely means that one stage of the lottery is degenerate. Uncertainty resolves either in the first stage or in the second stage, but not both. "One-shot early" resolution occurs when information resolves all at once in the first stage. This is a unique lottery, $(p, q, r)=(0.5,0,1)$, as shown in Figure II] With probability $p=0.5$, the individual sees bad news and updates his posterior to $q=0$, knowing for sure he will win the low prize in Stage 2. With the remaining probability $1-p=0.5$, he sees good news and updates to $r=1$, knowing for sure that he will win the high prize.

\footnotetext{
${ }^{9}$ We use this simplifying constraint in the experimental protocol, as well.
} 


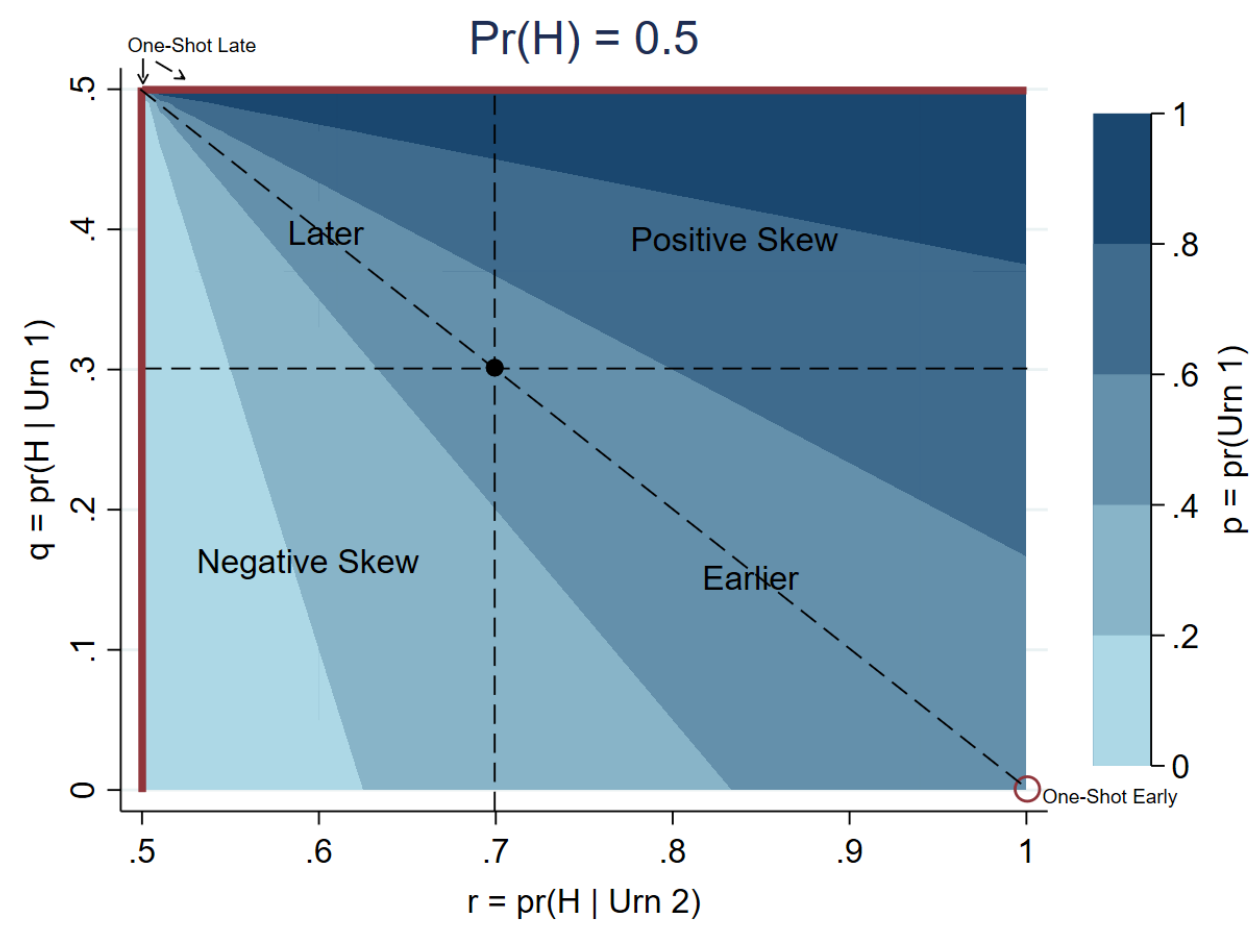

Figure II: Space of Actuarially Equivalent Lotteries. Given any lottery in $(p, q, r)$ space, points to the northwest are said to resolve uncertainty later, while points to the southeast resolve uncertainty earlier. One-shot late resolution corresponds to $q=0.5$ and/or $r=0.5$, as depicted on the north-most and west-most boundaries. One-shot early resolution corresponds to $q=0$ and $r=1$, in the southeast corner. $p>0.5$ is positively skewed while $p<0.5$ is negatively skewed.

"One-shot late" resolution is when uncertainty resolves all at once in the second stage, or when the first stage is degenerate. In one-shot late resolution, Stage 1 of the lottery reveals no information; regardless of the Stage 1 outcome, the individual will again face posterior $q, r=\theta=0.5$. This occurs any time $q$ or $r=0.5$. Either $p=0$, meaning $r=0.5$, shown on the left edge of the graph in Figure II] or $p=1$ and $q=0.5$, shown on the top edge of the graph in Figure II] If $q=r=0.5$, any $p \in[0,1]$ constitutes a one-shot late resolving lottery, which corresponds to the northwest corner of Figure II

These two cases encompass all possible one-shot resolution. A gradually-resolving lottery is, therefore, any other $(p, q, r)$. These gradually-resolving lotteries include any point in the interior of Figure II] as well as points along the bottom and right boundaries, excluding the corner points.

EARLY VS. LATE RESOLUTION. - It's easy to see that the earliest possible resolution of uncertainty is "oneshot early," when all uncertainty resolves in the first stage. Similarly, the latest possible resolution of uncertainty is "one-shot late," when all uncertainty resolves in the second stage. Beyond this, the ranking of lotteries follows the standard notion of Blackwell dominance. In the framework of two-stage lotteries, a Blackwell more informative lottery will have posteriors that are a mean-preserving spread of the posteriors under 
the Blackwell less informative lottery. Intuitively, earlier resolution of uncertainty involves signals that are more precise; good news is "better," with a posterior closer to 1, and bad news is "worse," with a posterior closer to 0 , in a lottery that resolves uncertainty earlier. Therefore, Lottery $\mathrm{A}$ is said to resolve uncertainty earlier than Lottery B if $q_{A}<q_{B}$ and $r_{A}>r_{B}{ }^{10}$ Thus, in Figure II any point to the southeast of another is Blackwell more informative and any point to the northwest is Blackwell less informative.

It is also helpful to define a symmetric lottery in our environment. Two lotteries are considered symmetric if they are symmetric around the $p=0.5$ diagonal. Symmetric lotteries are equally informative, but one reveals more information about the high state while the other reveals more information about the low state. Lotteries $\mathrm{A}$ and $\mathrm{B}$ are symmetric if $q_{A}+r_{B}=1$ and $q_{B}+r_{A}=1$, such as lotteries $(0.25,0.2,0.6)$ and $(0.75,0.4,0.8)$.

SKEWNESS. - Intuitively, in a positively-skewed information structure or compound lottery, seeing good news resolves more uncertainty about the high state than seeing bad news resolves about the low state. Positivelyskewed lotteries give good news less frequently, but, conditional on seeing good news, the decision maker can be more sure of the high state. Negatively-skewed lotteries resolve more uncertainty about the low state. They give bad news less frequently, but, conditional on seeing bad news, the decision maker can be more sure of the low state.

While notions of skewness are not absolute, we consider a choice of a lottery with $p>0.5$ to be "positively skewed." There exists a symmetric lottery with $p<0.5$ which is equally informative but negatively skewed. It could be that other lotteries exist which are "more positively skewed," but we compare within symmetric, equally-informative lotteries to identify positive or negative skewness.

Another implication of positive skew is that the lottery gives a higher posterior probability of winning regardless of the first stage outcome. That is, if Lotteries A and B are symmetric and therefore equally Blackwell informative, $q_{A}>q_{B}$ and $r_{A}>r_{B}$ indicates that Lottery A is more positively skewed than Lottery B. This also exactly corresponds to $p_{A}>p_{B}$. In a positively skewed lottery, it's more likely that the first stage will reveal "bad news," but conditional posteriors are higher than in a negatively skewed lottery.

\section{Information Structures}

The framework of the information structure treatment maps one-to-one into the compound lottery framework. In an information structure, the state of the world is determined at time $t=0$, giving rise to the high prize with probability $\operatorname{pr}(H)=\theta$ and otherwise the low prize. At time $t=1$, a signal is revealed about the state of the world. We reveal the signal in the following way. There are two urns, Urn 1 and Urn 2 , each of which can

\footnotetext{
${ }^{10}$ We consider a lottery to weakly Blackwell dominate another if one of these is a strict inequality while the other is weak. When discussing early or late choices, however, we will consider strict Blackwell dominance.
} 
contain some red and blue balls. We select and reveal a ball from either Urn 1 or Urn 2 . If the state is low, we reveal a ball from Urn 1 . If the state is high, we reveal a ball from Urn 2 . At time $t=2$, we reveal the urn from which the signal was drawn, and therefore reveal the prize won.

We can model this information structure analogously to the two-stage lotteries above. The information structure reveals a blue signal with probability $p$ and a red signal with probability $1-p$. Conditional on seeing a blue ball, the probability it came from Urn 2 , the winning urn, is given by $q$ and the probability it came from Urn 1 is $1-q$. Conditional on seeing a red ball, the probability it came from Urn 2 is given by $r$, and the probability it came from Urn 1 is given by $1-r$.

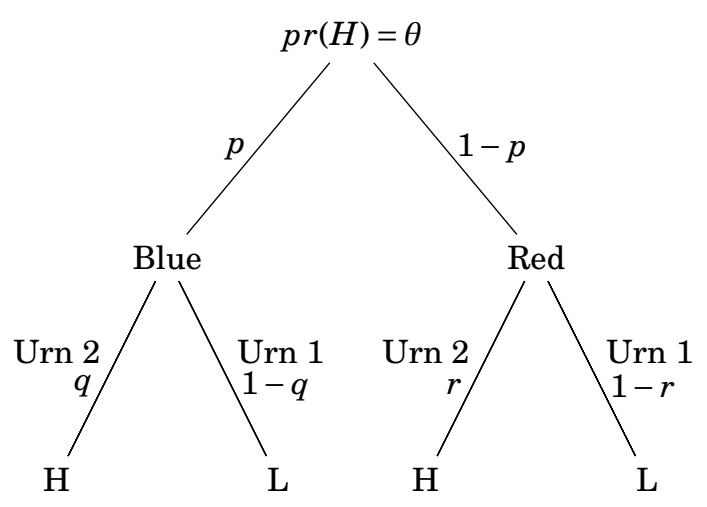

Figure III: Information Structure

Again we focus on situations where $q \leq \theta$ and $r \geq \theta$. Thus, identical to the two-stage lotteries, with probability $p$ the information structure reveals "bad news" in the form of a blue signal, and the posterior is reduced from $\theta$ to $q$. With probability $1-p$ the information structure reveals "good news" in the form of a red signal, and the posterior increases to $r$.

Analogous to the lottery framework, one-shot resolution of uncertainty corresponds to either a perfectly informative or perfectly uninformative signal. With one-shot early resolution, all uncertainty resolves in Stage 1 of the lottery in Figure III This is a perfectly informative signal, where the signal color exactly reveals the urn from which it was drawn, and therefore perfectly reveals the state of the world. With oneshot late resolution, all uncertainty resolves in Stage 2. This is a perfectly uninformative signal, where the signal color reveals nothing about the urn from which the signal was drawn. Gradual resolution corresponds to an informative signal that does not perfectly reveal the state of the world. Earlier resolution corresponds to a more informative signal and later resolution corresponds to a less informative signal, again following the standard Blackwell ordering. A signal from a skewed information structure can be interpreted in the same way as a skewed lottery.

While the Lottery and Information Structure treatments are analogous, and as similar as possible in our experimental design, there is one key difference between the two environments. In a two-stage lottery, the 
state of the world is determined at $t=2$. Uncertainty about the state can resolve beforehand, but the state is not determined until the ball is drawn from the urn. On the other hand, in an information structure, the state is determined at $t=0$. The signal can reveal more or less information about the state at $t=1$, but the state already has been decided. While theoretically identical, evidence from the psychology literature suggests individuals may view uncertainty differently when it's been resolved versus when it's not yet determined (Rothbart and Snyder, 1970). This gives reason to suggest individuals may have different preferences over analogous lotteries and information structures.

\section{EXPERIMENTAL DEsign}

We use a between-subject design, one treatment presenting choices over two-stage lotteries and the second presenting choices over information structures. Subjects make choices from various convex budget sets in $(p, q, r)$ space, constructing their most preferred two-stage lottery or information structure. The two environments are theoretically equivalent and are nearly identical in our experimental design. For ease of exposition, we detail the experimental design for the Lotteries treatment. The Information treatment differs as minimally as possible while changing the description from a two-stage lottery to a signal about the outcome of a predetermined lottery, according to the framework we describe above. Both sets of experiment instructions can be found in the Appendix.

\section{Lottery Protocol}

We present the environment to subjects just as we describe in Section III. Subjects are told that they will be participating in a lottery, and the lottery will take place in two steps: In Step 1, we select either Urn 1 or Urn 2 according to $(p, 1-p)$. Each of the urns could contain some number of red and blue colored balls ${ }_{11}^{11}$ In Step 2 , we randomly select one ball from the urn chosen in Stage 1 . If the ball drawn is red, the subject wins $\$ 11$ from the lottery. If the ball drawn is blue, the subject earns $\$ 2$. Thus, the subject faces uncertainty about the outcome of the lottery (e.g. whether the ball would be red or blue), but seeing the chosen urn after Step 1 may give some additional information about what the outcome will be. The subjects' task is to choose the composition of Urn 1 and Urn 2, as well as the likelihood of selecting Urn 1 vs. Urn 2. In other words, subjects are exactly choosing $(p, q, r)$. Subjects make these decisions for various "Lottery Sets," described below, which placed restrictions on the ranges of $(p, q, r)$.

To make the context easier for subjects to understand, we present the decision variables in a concrete and visible way. The Appendix shows an image of the subjects' screen during the experiment. Screens have 3

\footnotetext{
${ }^{11}$ The urns and balls were computerized, and all selections were made based on computerized random number generators.
} 
sliders, one corresponding to the relative likelihood of selecting Urn 1 vs. Urn 2 ( $p$ ), one corresponding to the proportion of red and blue balls in Urn $1(q)$, and the final corresponding to the proportion of red and blue balls in Urn $2(r)$. Subjects adjust the sliders in order to choose $p, q$, and $r$. The screens also contain 3 "Auto" buttons, one attached to each slider. Subjects are told, and the interface enforces, that they need to put one slider on Auto at all times. This slider will then adjust automatically to maintain $p q+(1-p) r=0.5$ while they are free to adjust the other two sliders. They also are free to change which slider was on "Auto" at any time. Each slider shows an equivalent color-coded pie chart that visually displays the selected probabilities and updates in real-time as subjects adjust the sliders 12

\section{Choice Sets}

We design 9 choice sets to disentangle preferences for one-shot, early, and skewed resolution. In some sets, the ranges of $q$ and $r$ are restricted to prevent one-shot early or one-shot late resolution. In others, we place restriction on skewness 13 We randomize the order of the choice sets in presenting them to subjects.

\begin{tabular}{|c|c|c|c|c|}
\hline & $\begin{array}{c}\mathbf{p} \\
{\left[p_{\min }, p_{\max }\right]}\end{array}$ & $\begin{array}{c}\mathbf{q} \\
{\left[q_{\min }, q_{\max }\right]}\end{array}$ & $\begin{array}{c}\mathbf{r} \\
{\left[r_{\min }, r_{\max }\right]}\end{array}$ & Description \\
\hline Set 1 & {$[0,1]$} & {$[0,0.5]$} & {$[0.5,1]$} & Unrestricted \\
\hline Set 2 & {$[0.5,1]$} & {$[0,0.5]$} & {$[0.5,1]$} & Only positively skewed \\
\hline Set 3 & {$[0,0.5]$} & {$[0,0.5]$} & {$[0.5,1]$} & Only negatively skewed \\
\hline \multicolumn{5}{|c|}{ Prevent One-Shot Early Resolution: } \\
\hline Set 4 & {$[0,1]$} & {$[0.2,0.5]$} & {$[0.5,0.8]$} & Symmetrically prevent early resolution \\
\hline Set 5 & {$[0,1]$} & {$[0.1,0.5]$} & {$[0.5,0.8]$} & Asymmetrically prevent early resolution (-) \\
\hline Set 6 & {$[0,1]$} & {$[0.2,0.5]$} & {$[0.5,0.9]$} & Asymmetrically prevent early resolution $(+)$ \\
\hline \multicolumn{5}{|c|}{ Prevent One-Shot Late Resolution: } \\
\hline Set 7 & {$[0,1]$} & {$[0,0.3]$} & {$[0.7,1]$} & Symmetrically prevent late resolution \\
\hline Set 8 & {$[0,1]$} & {$[0,0.3]$} & {$[0.6,1]$} & Asymmetrically prevent late resolution (-) \\
\hline Set 9 & {$[0,1]$} & {$[0,0.4]$} & {$[0.7,1]$} & Asymmetrically prevent late resolution (+) \\
\hline
\end{tabular}

Table II: Lottery Sets used in the experiment. "Asymmetrically prevent early resolution (+)" indicates that the earliest possible resolution was positively skewed, while "Asymmetrically prevent...(-)" indicates that it was negatively skewed, and similar for late resolution.

Set 1 allows for unrestricted choices—subjects can choose any $(p, q, r)$ such that $p q+(1-p) r=0.5$. Sets 2 and 3 force choices to be either positively or negatively skewed by requiring $p>0.5$ and $p<0.5$, respectively. Sets 4-6 prevent one-shot early resolution by requiring $q<0$ and $r<1$. These allow us to see next-best choices for individuals who choose one-shot early resolution ( $q=0$ and $r=1)$ in Set 1 . These sets differ in the skewness of the earliest possible resolving lottery—in Set 4, the earliest resolving lottery is unskewed,

\footnotetext{
${ }^{12}$ The sliders start in a random position which differs across the various sets. The default Auto slider is also randomly determined in each set.

${ }^{13}$ In practice, sliders in the restricted sets simply display a restricted range, but subjects' choices are presented nearly identically to the unrestricted set.
} 
while it's negatively skewed in Set 5 and positively skewed in Set 6. Similarly, Sets 7-9 prevent one-shot late resolution by requiring $q<0.5$ and $r>0.5$. These allow us to see next-best choices for individuals who choose one-shot late resolution in Set 1 . The three sets again differ in the skewness of the latest possible resolving lottery.

It's important to see how and why these sets allow us to identify and disentangle preferences for one-shot late resolution and preferences for early/late resolution. A subject who chooses one-shot resolution of uncertainty in Set 1 necessarily chooses either the earliest or the latest possible resolution. Thus, it's impossible to say whether he expresses a preference for one-shot resolution or a preference for early/late resolution. The additional sets allow us to do just that. A subject who chooses one-shot early resolution in Set 1 in order to express preferences for one-shot information will switch to choosing one-shot late resolution in Set 4, for example, since one-shot late resolution is the only available one-shot information in this set. However, a subject who chooses one-shot early resolution in Set 1 in order to express a preference for early resolution will choose the earliest possible resolution in Set 4 ( $q=0.2$ and $r=0.8$ ), regardless of the fact that it's now gradually resolving.

In addition to the 9 sets listed in Table II] subjects answer 3 price-list questions comparing different lotteries. These questions serve to elicit the amount of money a subject requires to switch from one lottery to another. We will use these questions to measure strength of preference and rule out the explanation that subjects were simply indifferent over all lotteries.

In the price-list questions, we present subjects with two lotteries, one denoted "Option A" and the other "Option B." Then, we ask subjects to choose between Option A and Option B 21 times, where each question adds a nominal payment on top of either Option A or Option B, as shown below. A subject who is indifferent between Option A and Option B will always choose the one which gives additional payment. A subject who strictly prefers Option A, for example, would be willing to forgo this additional payment in order to receive Option A over Option B. In one price list, Option A is one-shot early resolution and Option B is one-shot late resolution. In the other two price lists, we design Option A and Option B to test between early/late preference and skewness. 


\begin{tabular}{lcl}
\multicolumn{3}{l}{ Would you rather have... } \\
Option A $+\$ 0.50$ & or & Option B \\
Option A $+\$ 0.45$ & or & Option B \\
Option A $+\$ 0.40$ & or & Option B \\
$\vdots$ & $\vdots$ & $\vdots$ \\
Option A +\$0.05 & or & Option B \\
Option A & or & Option B \\
Option A & or & Option B $+\$ 0.05$ \\
$\vdots$ & $\vdots$ & $\vdots$ \\
Option A & or & Option B $+\$ 0.40$ \\
Option A & or & Option B $+\$ 0.45$ \\
Option A & or & Option B $+\$ 0.50$
\end{tabular}

Admittedly, our experimental design is fairly complicated. However, theoretical models predict preferences over the entire domain of lotteries or information structures. For example, a preference for one-shot resolution implies that a one-shot lottery is preferred to any gradually resolving lottery. This is impossible to see with binary choices-one would have to compare one-shot resolution to every possible gradually resolving lottery. In order to test the theories rigorously, we need to be able to see whether one-shot resolution is chosen among the entire set of actuarially-equivalent lotteries.

To mitigate the trade-offs between simplicity and rigor, we give many examples along with the instructions. We use a screen-captured video to demonstrate how to adjust the sliders and how the lotteries change as a result. Additionally, we have a number of practice rounds and corresponding comprehension questions. The price list questions also help us to identify subjects' understanding, as we can compare willingness-topay in binary choices to choices in the main experiment. Furthermore, we give subjects a non-incentivized questionnaire to get a picture for how well they understand the experiment setup and how they describe their own preferences. We use this as suggestive evidence toward their understanding of the experimental design, and we present this data at the end of the Results section.

\section{Timeline}

Studying preferences over uncertainty resolution necessarily requires a time delay between the two stages of resolution. However, having subjects wait a significant amount of time, on the order of days or weeks, could introduce instrumental information concerns, as subjects might prefer to know their future earnings for planning purposes, for example. We avoid this by keeping subjects in the lab for the entire duration of resolution. To balance these concerns, we implement a fairly substantial but experimentally-controlled time delay in the lab of approximately 30 minutes between the two stages of resolution 14

\footnotetext{
${ }^{14} 30$ minutes certainly pales in comparison to the years spent waiting to know whether or not one will develop a health condition, for example, so we view this as a lower bound on preferences. Masatlioglu et al. (2017) also use a duration of approximately 30 minutes
} 


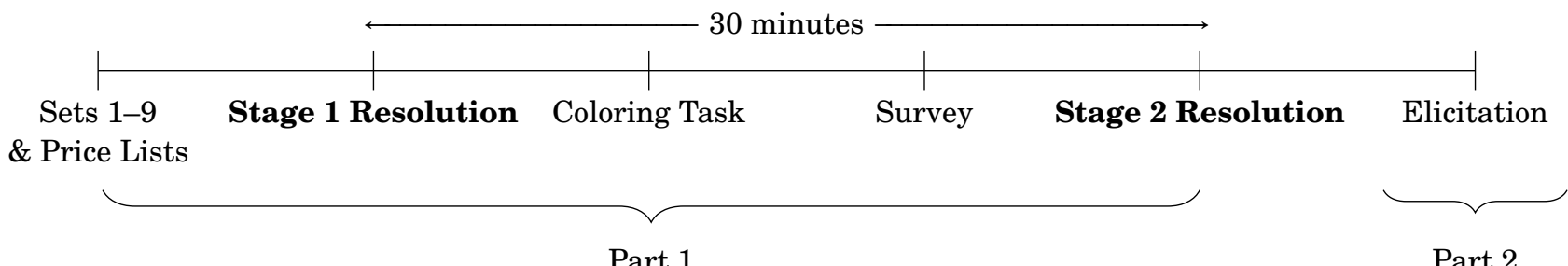

Figure IV: Timeline of experiment

We refer to the lottery task and related events collectively as Part 1. After reading aloud instructions for all of Part 1, subjects participate in a number of practice rounds to familiarize themselves with the computer and slider mechanics. At this time, they answer comprehension questions to ensure they understand the task. They are not able to proceed without answering the questions correctly. Following this, they choose their most preferred lottery in Sets 1-9 and in the price list elicitations. Once all subjects have finished, the computer randomly selects one set or price list and reveals the first stage outcome (Stage 1 Resolution) 15 Thus, subjects are told whether the ball will be drawn from Urn 1 or Urn 2. They are shown the composition of that urn and are reminded that the ball will be drawn at the end of Part 1.

After the Stage 1 resolution, subjects participate in a "Coloring Task" and a non-incentivized survey. The primary purpose of these tasks is to create a time delay between Stage 1 and Stage 2 resolution. In addition, the coloring task gives subjects a visualization of the urn and probabilities of winning the high and low prize, and aims to make lingering uncertainty (or lack thereof) more salient. We remind subjects of the red and blue ball composition of the selected urn and then subjects use their computers to "color" an urn of those proportions. Their screens display 100 numbered balls and they click each ball to turn it red or blue according to the proportions of their actual urn. That is, if their chosen urn contains $30 \%$ red balls and $70 \%$ blue balls, they would color balls numbered 1-30 red and balls 31-100 blue ${ }^{16}$ See the Appendix for an image of the subjects' screen during the Coloring Task. The survey asks various hypothetical lottery questions as well as questions on personal preferences and demographics. After all subjects complete the Coloring Task and survey, we draw the seleted ball from the urn and reveal it to subjects (Stage 2 Resolution). Approximately 30 minutes pass between Stage 1 Resolution and Stage 2 Resolution, and subjects know ahead of time that

and find significant willingness to pay for information, so we expected 30 minutes to be a reasonable wait duration and this allowed us to compare our results to theirs directly. The literature has not yet established whether preferences change with the duration of waiting. This is an interesting open question for future research.

${ }^{15}$ Section $\mathrm{G}$ in the Appendix confirms that this random problem selection mechanism does not interact with a preference for one-shot resolution.

${ }^{16}$ Subjects were told of this task in the initial instructions, so one purpose of the Coloring Task is to ensure subjects can't avoid thinking about the second stage of the lottery and that they know this in advance. Falk and Zimmermann (2016) demonstrate that attention is an important component of preferences, and we wanted to represent a situation where unresolved uncertainty was salient. In addition, the Coloring Task helped subjects visualize the abstract probabilities in the lottery. In Stage 2 resolution, the computer drew a random number between 1 and 100. This corresponds to one of the 100 colored balls, so subjects could easily see whether they had won the high or low prize. 
this will be the case.

At the beginning of the experiment, we tell subjects that there will be a short "bonus" Part 2. This consists of various elicitation questions from Dean and Ortoleva (2016). The questions are designed to measure risk aversion, present bias, time discounting, common ratio and common consequence violations, and violations of several axioms. Since the time preference elicitation questions involved future payments, all payments from Part 2 are paid through Venmo money transfers. See the Appendix for elicitation instructions and specific payment details.

\section{RESULTS}

We analyze data from a total of 182 subjects -69 subjects in the Lottery treatment, 73 in the Information treatment, and 40 in control sessions described in Section VI Subjects are primarily undergraduate students at the Ohio State University, recruited through ORSEE (Greiner, 2004). Sessions lasted approximately 90 minutes and payments averaged $\$ 19$, including a $\$ 10$ show-up fee. We conducted the experiment using zTree (Fischbacher, 2007). For two sample statistical comparisons, we use two-tailed Mann-Whitney ranksum tests. We confirm with ranksum tests of equality on the medians. For tests within sample, we use chi-square goodness of fit tests, and for within-subject tests we use two-sided sign tests.

\section{Early vs. Late}

Preferences along both the early vs. late dimension and preference for one-shot resolution predict that individuals will choose the earliest (one-shot early) or latest (one-shot late) resolution of uncertainty in the unrestricted set. Figure $\mathrm{V}$ reports the main results, presenting the percentage of subjects who choose one-shot early, one-shot late, and intermediate resolution in the unrestricted set in both treatments ${ }^{17}$ Graphs in Figure VI] in the Appendix plot all subjects' choices in $(p, q, r)$ space in both treatments.

In the Lottery treatment, we see an overwhelming majority, $70 \%$ of subjects, choosing intermediate resolution of uncertainty. These individuals do not take the opportunity to resolve all uncertainty in Stage 1 of the lottery, nor do they avoid information completely. They have available lotteries which are more informative and lotteries which are less informative, but they instead choose intermediate uncertainty resolution. In the Information treatment, subjects are divided. $49 \%$ of subjects choose a perfectly informative or perfectly uninformative information structure, with the remainder choosing intermediate resolution.

\footnotetext{
${ }^{17}$ We use the term "intermediate" to refer to choices which are not the earliest or latest resolution of uncertainty. We reserve the term "gradual" to contrast with one-shot resolution. Note that intermediate choices are always gradual, but gradual choices need not give intermediate resolution. For example, in preventing one-shot early resolution, the earliest resolution is now gradual but we do not call this "intermediate" since it is the choice of the earliest possible resolution.
} 


\section{Unrestricted Set Choices}

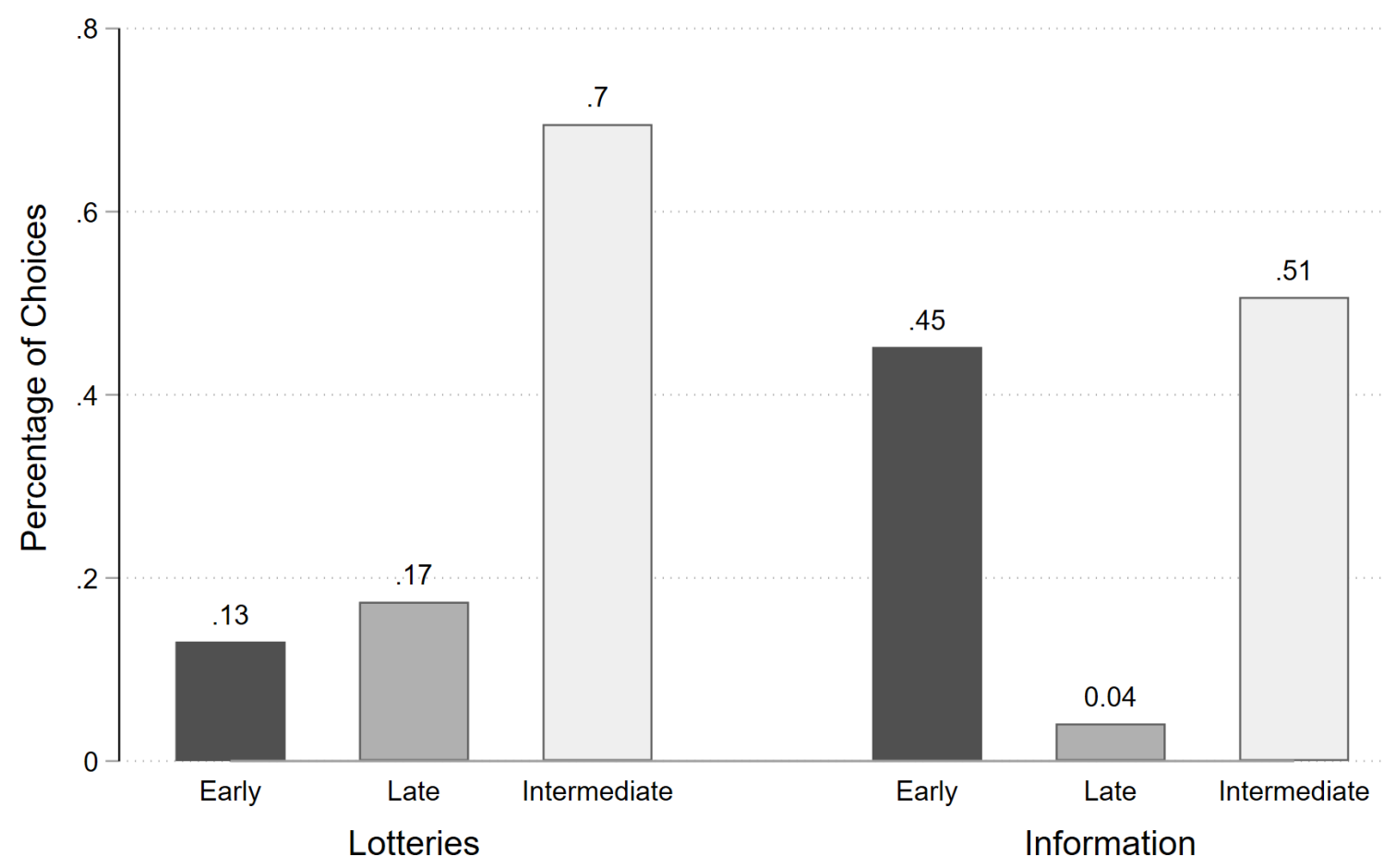

Figure V: Percentage of Early, Late, and Gradual Resolution Choices Across Treatments

As discussed in Section II] previous experiments studying preferences over the resolution of uncertainty and information acquisition primarily have compared early to late resolution. Generally these papers find a stronger preference for early, though this preference can change with lottery stakes (Ganguly and Tasoff, 2016), attention (Falk and Zimmermann, 2016), and appears more heterogeneous for outcomes which have not been determined (Zimmermann, 2015). However, all of these studies have been limited to discrete comparisons across lotteries or information structures. A benefit of our design is that it allows subjects to choose any uncertainty resolution. We find the majority of individuals do not prefer the earliest or latest resolution when given freedom to choose among all possibilities, and instead they prefer some type of intermediate resolution.

We now look within those who do choose the earliest or latest manner of uncertainty resolution to see whether preferences are more for information-seeking (early resolution) or information avoidance (late resolution). Of those who do choose a manner of one-shot resolution in the Lottery treatment, we find no significant difference in the percentages of choices- $13 \%$ of subjects prefer to resolve all uncertainty in the first stage of the lottery, while $17 \%$ prefer to resolve all uncertainty in the second stage $(p=0.517)$. Of subjects who choose early or late resolution in the Information treatment, however, we see a very strong and significant preference for early over late resolution. $45 \%$ of subjects chose a perfectly informative signal to resolve all uncertainty, 
while only $4 \%$ chose to resolve no uncertainty with a perfectly uninformative signal $(p<0.0001) 18$

We find the same general trends in the restricted sets, as well. Graphs VI $[$ XIV] in the Appendix plot all set choices in $(p, q, r)$ space in both treatments. Table III gives the percentage of individuals, per treatment, choosing the earliest and latest possible resolution in each set ${ }^{19}$ The percentages denoted with the dagger symbol indicate that the earliest or latest resolution is one-shot, while the others are gradually-resolving. In both treatments, we see a majority of subjects choosing neither the earliest nor the latest resolution, instead favoring something intermediate. Among those who do choose one of the extremes in the Lottery treatment, we see a preference for late resolution. The preference holds directionally in 7 of 9 sets, and the difference is significant in three sets. On the other hand, information structures show a clear preference for early over late resolution. On average, over one third of individuals choose the most informative signal structure in the Information treatment in each set, with very few individuals choosing completely uninformative signals.

Tables VIII and IX in the Appendix compare the percentages of early, late, and gradual resolution by treatment. The between-treatment results present a similar picture to the within-treatment conclusions. In each set, a significantly higher percentage of individuals choose early resolution in the Information treatment compared to the Lottery treatment $(p<0.0053)$. A higher percentage of individuals choose late resolution in the Lottery treatment compared to the Information treatment, and this difference is highly significant in 7 of 9 sets $(p<0.0128)$ and marginally significant in one $(p=0.0736)$. Results clearly indicate a stronger preference for early resolution in the Information treatment, and a stronger preference for late resolution in the Lottery treatment.

These results suggest that we are more likely to see information avoidance in environments more similar to the Lottery treatment. When the outcome has been determined and is theoretically discoverable, individuals are more willing to seek out information on this event. When the outcome has yet to be determined, individuals avoid information and delay uncertainty resolution.

Result 1. The majority of individuals prefer intermediate resolution in both treatments. Among those who don't, there is a clear preference for early resolution in the Information treatment and a preference for late resolution in the Lottery treatment.

\footnotetext{
${ }^{18}$ Given that our design offers a continuum of choice, it's possible that the subjects choosing gradual resolution would express a preference opposite the general comparison between one-shot early and one-shot late. For example, in the Information treatment, it's possible that the the remaining $51 \%$ of subjects choosing gradual resolution would prefer one-shot late over one-shot early. In Section VI we present results from the binary choice price lists, and show that this isn't the case.

${ }^{19}$ We classify a subject as choosing early information if he chooses the Blackwell most informative resolution $\left(q=q_{\min }\right.$ and $\left.r=r_{\text {max }}\right)$ and we classify a subject as choosing late information if he chooses the Blackwell least informative resolution $\left(q=q_{\max }\right.$ and $\left.r=r_{\min }\right)$.
} 


\begin{tabular}{|c|c|c|c|c|c|c|}
\hline \multirow[b]{2}{*}{ Set } & \multicolumn{3}{|c|}{ Lotteries } & \multicolumn{3}{|c|}{ Information } \\
\hline & Earliest & Latest & p-value & Earliest & Latest & p-value \\
\hline 1 & $13.04^{\dagger}$ & $17.39^{\dagger}$ & 0.5127 & $45.21^{\dagger}$ & $4.11^{\dagger}$ & 0.0000 \\
\hline 2 & $10.14^{\dagger}$ & $30.43^{\dagger}$ & 0.0082 & $35.62^{\dagger}$ & $6.85^{\dagger}$ & 0.0002 \\
\hline 3 & $10.14^{\dagger}$ & $20.29^{\dagger}$ & 0.1266 & $41.10^{\dagger}$ & $2.74^{\dagger}$ & 0.0000 \\
\hline \multicolumn{7}{|c|}{ Prevent One-Shot Early Resolution: } \\
\hline 4 & 5.8 & $34.78^{\dagger}$ & 0.0002 & 46.58 & $15.07^{\dagger}$ & 0.0006 \\
\hline 5 & 10.14 & $20.29^{\dagger}$ & 0.1266 & 31.51 & $10.96^{\dagger}$ & 0.0071 \\
\hline 6 & 4.35 & $20.29^{\dagger}$ & 0.0076 & 32.88 & $9.59^{\dagger}$ & 0.0023 \\
\hline \multicolumn{7}{|c|}{ Prevent One-Shot Late Resolution: } \\
\hline 7 & $5.80^{\dagger}$ & 13.04 & 0.1655 & $31.51^{\dagger}$ & 1.37 & 0.0000 \\
\hline 8 & $13.04^{\dagger}$ & 10.14 & 0.6171 & $36.99^{\dagger}$ & 0 & - \\
\hline 9 & $13.04^{\dagger}$ & 11.59 & 0.8084 & $32.88^{\dagger}$ & 1.37 & 0.0000 \\
\hline
\end{tabular}

Table III: Percentage of Early and Late Choices in Each Set. Values with a dagger indicate one-shot resolution. p-values are from a one-sample chi-square goodness-of-fit test comparing the choice of early vs. late, with the null hypothesis that the percentages of early and late choices are $50 \%$.

\section{One-Shot vs. Gradual}

In addition to the observed preference for early information and late-resolving lotteries, the next question is whether there is any evidence of a preference for one-shot resolution in either treatment. Recent theories characterize individuals who prefer uncertainty to resolve all at once (Palacios-Huerta, 1999, Köszegi and Rabin, 2009, Dillenberger, 2010) or dislike one-shot resolution of uncertainty (Ely et al., 2015). We use choices in the restricted sets to analyze whether individuals express a preference for or against one-shot resolution of uncertainty.

First, we refer back to Table [III, which allows us to see the percentage of one-shot choices in each set, by treatment. In the Lottery treatment, one-shot resolution is far from prevailing-only $30 \%$ of individuals choose one-shot resolution in the unrestricted set. Across all sets, nearly two thirds of subjects in the Lottery treatment choose a type of gradual resolution instead of resolving uncertainty all at once. One-shot choices are most common in Sets 1-3, where both one-shot early and one-shot late are available, but a significant majority of subjects choose a lottery with gradual resolution in all but Set $2(p<0.0115, p=0.1176$ for Set 2$)$. One-shot resolution is more common in the Information treatment, but still constitutes the minority choice. Just shy of 50\% of individuals choose one-shot resolution in the unrestricted set, and the other sets lie strictly below this percentage. There is no significant difference in the percentages of one-shot versus gradual choices in Sets $1-3$, when both one-shot early and one-shot late are available $(p>0.1979)$, but gradual resolution is significantly more common in the remaining 6 sets $(p<0.0262)$. On the whole, aggregate statistics conclude that gradual resolution generally is preferred to one-shot resolution in both treatments.

As discussed, preference for one-shot resolution can be confounded by a preference for early or late infor- 
mation. Though we see gradual resolution more preferred than one-shot, aggregate choices could be reflecting individuals with strong early/late preferences which overshadow the nuances of one-shot resolution. We delve deeper into the preference for one-shot resolution by looking to see whether individuals choose the earliest or latest resolution more frequently when it's one-shot compared to when it's gradual. That is, we compare the average percentage of individuals who choose early resolution when it's one-shot (Sets 1-3 and Sets 7-9) to the average percentage of individuals who choose the earliest possible resolution when it is gradual (Sets 4-6). Similarly, we compare the average percentage of individuals choosing one-shot late resolution (Sets 1-3 and Sets 4-6) to the average percentage of individuals who choose the latest possible resolution when it is gradual (Sets 7-9).

\begin{tabular}{lccc}
\hline & One-Shot & Gradual & p-value \\
\hline Lotteries & & & \\
\hline Earliest & 10.87 & 6.76 & 0.0192 \\
Latest & 23.91 & 11.59 & 0.0002 \\
\hline Information & & & \\
\hline Earliest & 37.21 & 36.99 & 1.000 \\
Latest & 8.22 & 0.91 & 0.0000 \\
\hline
\end{tabular}

Table IV: Average percentage of early and late choices when early/late is one-shot vs. gradual

For lotteries, we see a marginally significantly higher percentage of choices for early resolution when oneshot. About $11 \%$ of individuals choose early resolution when one-shot early is available, compared to $7 \%$ when the earliest resolution is gradual (two-sided sign test $p=0.0192$ ). We see a much larger and significantly higher percentage of individuals choosing late-resolving lotteries when they are able to learn nothing in the first stage resolution compared to when latest resolution reveals some information $(23.91 \%$ vs. $11.59 \%$, $p=0.0002)$. These individuals take the opportunity to avoid all information when possible, but this doesn't translate to late resolution when complete information avoidance is unavailable. This suggests that some individuals do have a preference to resolve all or no uncertainty in stage one of the lottery-both early and late resolution are more appealing in lotteries when they resolve uncertainty all at once.

For information structures, we find no significant difference in the percentage of individuals choosing early resolution. Roughly $37 \%$ of individuals choose the earliest possible resolution, regardless of whether it's one-shot or gradual. We've already established a strong preference for early resolution in the Information treatment, and this seems to overwhelm any one-shot preference. These individuals simply want to learn as much as possible, regardless of whether the information is one-shot or gradual. On the other hand, we see significantly more choices of late-resolving information when late resolution is one-shot (8.22\% vs. $0.91 \%$, $p=0.0000$ ). This suggests that some individuals prefer to delay all uncertainty resolution when possible in 
the Information treatment, but late resolution is less appealing when forced to acquire some information.

WITHIN-SUBJECT ANALYSIS. - Finally, we turn to within-subject analysis and exploit the main purpose of the different sets used in our experimental design. While we see suggestive evidence that early and late resolution are more appealing when one-shot, the question still remains as to whether individuals prefer any type of oneshot resolution to any gradually resolving lottery. If this were the case, we would see individuals transition from choosing one-shot early to one-shot late when one-shot early is unavailable, and vice versa. The model of Dillenberger (2010) assumes individuals are indifferent between one-shot early and one-shot late resolution, but that they prefer these to any gradual resolution. Kosszegi and Rabin (2009) predict that individuals weakly prefer one-shot early over one-shot late resolution, but one-shot late is still preferred to gradual resolution of uncertainty. Both these models predict that individuals will choose one-shot early resolution when one-shot late is unavailable, and will choose one-shot late resolution when one-shot early is unavailable.

As shown in Figure V, we see $13 \%$ of individuals choosing one-shot early resolution in the unrestricted Lottery set and $45 \%$ of individuals choosing one-shot early resolution in the unrestricted Information structure set. We now focus our within-subject analysis on these individuals-if they have a persistent preference for one-shot resolution, they will switch to choosing one-shot late resolution in Sets 4-6 when one-shot early resolution is unavailable. If they have a persistent preference for early resolution, they will continue choosing the earliest resolving lottery, even when it gives partial information.

\begin{tabular}{lcccc}
\hline & Earliest & Latest & Other & \\
\hline Prevent OS Early & & & & \\
\hline Lotteries & $44.44 \%$ & $22.22 \%$ & $33.33 \%$ & $\mathrm{n}=9$ \\
Information & $72.73 \%$ & $9.09 \%$ & $18.18 \%$ & $\mathrm{n}=33$ \\
\hline Prevent OS Late & & & & \\
\hline Lotteries & $0 \%$ & $25 \%$ & $75 \%$ & $\mathrm{n}=12$ \\
Information & $0 \%$ & $0 \%$ & $100 \%$ & $\mathrm{n}=3$ \\
\hline
\end{tabular}

Table V: Set 4 choices for those who chose one-shot early resolution in Set 1, and Set 7 choices for those who chose one-shot late resolution in Set 1 .

Looking at individuals who choose a one-shot early resolving lottery in Set 1, we see that very few Set 4 choices are consistent with a preference for one-shot resolution-only $22 \%$ of subjects switch to choosing oneshot late resolution in Set 4 where one-shot early is unavailable $44 \%$ of subjects choose lotteries consistent with a preference for early resolution, as they continue to choose the earliest possible resolving lottery. The remaining $33 \%$ of subjects choose neither the earliest nor the latest resolution when one-shot early is unavail-

\footnotetext{
${ }^{20}$ Given the low percentage of subjects choosing early resolution in the Lottery treatment to begin with, this is just two individuals.
} 
able. As a result, it seems the choice of one-shot early resolution in the unrestricted set is driven primarily by a preference for early resolution, if anything, rather than a preference for one-shot resolution. The same observation holds for choices in Sets 5 and 6 (see Appendix Table $\mathrm{X}$.

Looking at individuals who choose the earliest resolution in Set 1 of the Information treatment, results are even more striking. Again we see very few Set 4 choices consistent with a preference for one-shot resolutiononly $9 \%$ of subjects choose perfectly uninformative signals when a perfectly informative signal is unavailable. On the contrary, $73 \%$ of subjects continue to choose the most informative signal structure even when it gives gradual information, consistent with a preference for early resolution. Again, we see a similar choice pattern

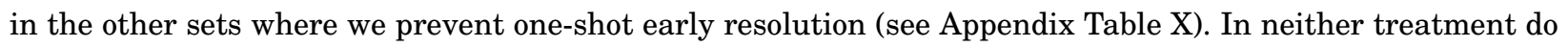
we see subjects substituting one-shot late resolution for one-shot early resolution, contrary to the predictions of Köszegi and Rabin (2009) and Dillenberger (2010).

Turning to a preference for late resolution, we focus on the $17 \%$ of individuals in the Lottery treatment and $4 \%$ of individuals in the Information treatment who chose one-shot late resolution in Set 1 , which are admittedly small samples. If these individuals prefer one-shot resolution, they will switch to choosing oneshot early resolution when one-shot late resolution is unavailable.

Contrary to the predictions of a preference for one-shot resolution, we find no subjects in either treatment transitioning to choosing one-shot early resolution in Set 7, where one-shot late is unavailable. $25 \%$ of individuals in the Lottery treatment continue to choose the latest-resolving lottery, suggesting their preference primarily is for late information. A similar pattern holds in Sets 8 and 9.

Overall, we see very little evidence, in either treatment, of a preference for one-shot resolution of uncertainty. $30 \%$ of individuals choose a one-shot resolving lottery when choices are unrestricted, but very few demonstrate choices in other sets consistent with a preference for one-shot resolution. Over 50\% of individuals choose a one-shot information structure in the unrestricted set, but this appears most strongly driven by a preference for early information. Previous studies on one-shot resolution (Zimmermann, 2015) have been restricted to comparing one-shot resolution with select gradual resolution. We find that, when given an expanded choice set, individuals do not consistently prefer one-shot over gradual resolution 21

Result 2. There is little evidence of a persistent preference for one-shot resolution of uncertainty in either the Lottery or Information treatments.

The models of Ely et al. (2015) predict a preference against one-shot resolution. Ely et al. (2015) model decision makers who derive utility from suspense or surprise 22 Suspense increases with increasing variance in

\footnotetext{
${ }^{21}$ In Section $\mathrm{E}$ in the Appendix, we analyze the axioms underlying the model in Dillenberger 2010 , to see whether it is the axioms or the predictions of the model which fail. We find few subjects satisfy all of the axioms in the theory (29\% in the Lottery treatment and $15 \%$ in the Information treatment), but these subjects also do not exhibit a preference for one-shot resolution.

${ }^{22}$ These models were primarily designed to analyze situations like sports games and mystery novels where we might expect individuals
} 
beliefs from one period to the next, and surprise increases with the difference in beliefs from one period to the next. The authors derive suspense- and surprise-optimal policies for general decision environments. Given our setup with two time periods, Ely et al. (2015) show that the lottery which maximizes suspense is the interior lottery $(0.5,0.15,0.85)$, and the lottery which maximizes surprise is the interior lottery $(0.5,0.25,0.75){ }^{23}$

Given an exact definition of suspense, $(0.5,0.15,0.85)$, we find that no subject in either treatment chooses the suspense-maximizing lottery. If we allow for some noise around the maximum, and consider any lottery with $q \in(0.10,0.20)$ and $r \in(0.80,0.90)$, we find that $6 \%$ of intermediate Lottery choices and $8 \%$ of intermediate Information choices in the unrestriced set maximize suspense $(p=0.74)$. Turning to surprise, with an exact definition of $(0.5,0.25,0.75)$, we find $2 \%$ of intermediate Lottery choices and $8 \%$ of intermediate Information choices maximize surprise $(p=0.20)$. With a less restrictive definition of $q \in(0.20,0.30)$ and $r \in(0.70,0.80)$, this increases to $4 \%$ and $11 \%$, respectively $(p=0.24$ ). Overall, under our more generous definitions, $10 \%$ of intermediate Lottery choices and 19\% of intermediate Information choices maximize suspense or surprise (betweentreatment difference $p=0.27){ }^{24}$ This accounts for a non-trivial proportion of the intermediate choices we see in the unrestricted set25

While we don't find overwhelming evidence for the specific predictions of the suspense and surprise models, we do find a high proportion of intermediate choices. In general, gradual resolution of uncertainty is more suspenseful and more surprising than one-shot resolution, so our results seem more in line with these motivations than with resolving uncertainty all at once. The hypothetical questions between Stage 1 and Stage 2 resolution give further suggestive evidence. We asked subjects to rate, on a scale from 1-7, how much they enjoyed mystery novels. We find those choosing gradual resolution in the Information treatment report higher enjoyment of mystery novels compared to those choosing one-shot resolution, an average rating of $5.47 \mathrm{vs.} 4.57$ $(p=0.029)$. Results are directionally similar in the Lottery treatment, though the difference is not significant (5.38 vs. $5.29, p=0.84)$.

\section{Skewness}

To analyze preferences over skewness, we focus on symmetric lotteries or information structures. With symmetric lotteries, neither is more informative after Stage 1 resolution, but the lotteries differ in the state

\footnotetext{
to have a strong preference for suspense and/or surprise. We can apply these models to our lottery environment, but do not believe this was intended to be their primary application.

${ }^{23}$ The suspense-maximizing lottery was available in Sets 1-3 and 7-9. The surprise-maximizing lottery was available in all Sets.

${ }^{24}$ All subjects categorized as choosing the suspense- or surprise-maximizing lottery under either definition express a positive willingness to pay on one of the price list questions. Thus, it is not the case that these subjects are simply choosing randomly and happened to choose an interior lottery corresponding to high suspense or surprise. These subjects have strict preference over information and reveal that their optimal choice is an interior lottery with high suspense or surprise.

${ }^{25}$ In Section $\mathrm{F}$ of the Appendix, we analyze the internal consistency of subjects' choices across different lottery sets. We find that subjects choosing suspense- or surprise-maximizing lotteries are no less consistent than other subjects.
} 
of the world about which they resolve more uncertainty. Positively skewed lotteries resolve more uncertainty about the good state of the world while negatively skewed lotteries resolve more uncertainty about the bad state. We consider an individual to choose a positively skewed lottery when he could have chosen the symmetric and equally-informative negatively skewed lottery, and vice versa. This focuses our analysis on interior choices which do not lie on the diagonal.

\begin{tabular}{lccc}
\hline & Lotteries & Information & p-value \\
\hline Unrestricted & & & \\
\hline Positively Skewed & $48.84 \%$ & $45.83 \%$ & 0.82 \\
p-value & 0.8788 & 0.6831 & \\
\hline Symmetrically Prevent Early & & & \\
\hline Positively Skewed & $41.18 \%$ & $41.67 \%$ & 0.60 \\
p-value & 0.3035 & 0.5637 & \\
\hline Symmetrically Prevent Late & & & \\
\hline Positively Skewed & $51.02 \%$ & $72.22 \%$ & 0.019 \\
p-value & 0.8864 & 0.0077 & \\
\hline
\end{tabular}

Table VI: Percentage of subjects expressing choice of positively skewed lottery or information structure.

For lotteries, we see no significant preference for or against positively skewed resolution. Regardless of how choices were restricted, there is still an equal percentage of individuals choosing positively and negatively skewed lotteries. For information structures, on the other hand, we see a significant preference for positively skewed information when one-shot late resolution is restricted. This is in line with results from Masatlioglu et al. (2017) who show a preference for positively skewed information structures. Our results suggest, though, that this preference might not exist when all possible information structures are available. Masatlioglu et al. (2017) use binary choices, which is closer to a restricted set, but we don't see a significant preference for positive skew in the unrestricted set.

Finally, we look to see whether early and late resolution are more attractive when the earliest resolution is positively or negatively skewed. We find that, in the Lottery treatment, individuals slightly favor negative skew. $10 \%$ of subjects choose the earliest resolving lottery when it's negatively skewed compared to $4 \%$ when it's positively skewed ( $p=0.10$ ). This suggests that while individuals generally don't prefer early resolution in the Lottery treatment, the negative skew makes it slightly more attractive. On the other hand, we've seen that individuals have a stronger preference for late resolution in the Lottery treatment, and this does not interact with lottery skewness. $10 \%$ choose the latest resolving lottery when it's negatively skewed compared to $12 \%$ when it's positively skewed $(p=0.766)$. 
In the Information treatment, $32 \%$ of individuals choose the most informative information structure when it's negatively skewed compared to $33 \%$ who choose the most informative positively skewed information structure $(p=0.741) .0 \%$ choose the least informative information structure when it's negatively skewed and $1 \%$ choose the least informative information structure when it's positively skewed $(p=0.321)$. This again is consistent with results from Masatlioglu et al. (2017). They find that individuals prefer positively skewed information compared to negatively skewed information that is equally informative (as we conclude above), but the preference for early resolution dominates. In other words, there exists a lexicographic preferenceindividuals choose the earliest possible resolution, but among equally informative signals, they prefer positive skew.

Result 3. Individuals weakly prefer negative skew in the Lottery treatment and prefer positive skew in the Information treatment.

\section{Robustness}

Taken together, our results conclude that preferences over lotteries are significantly different from preference over information structures, despite their theoretical equivalence. In this section, we analyze the robustness of our main results. In particular, we turn to our willingness-to-pay elicitation to rule out the possibility that subjects are simply indifferent over all lotteries and information structures. In Section F in the Appendix, we look at the internal consistency of subjects' choices across sets, and we correlate choices with individual risk and time preferences measures in Section $\mathrm{D}$. In Section $\mathrm{G}$, we discuss issues of incentive compatibility.

\section{Indifference}

While we see consistent patterns of choices, the natural question is whether these result from strict preferences. Choices could not affect the state of the world- $\operatorname{pr}(H)=0.5$ in all lottery sets and information structures—so a pure expected utility maximizer would be indifferent among all alternatives.

As described in the Experimental Design, we present subjects with 3 price-list choices to get a rough picture of indifference ${ }^{26}$ In one price list, we explicitly ask for strength of preference between one-shot early and oneshot late resolution. The second price list captures the early/late dimension when both are gradually resolving lotteries. Option A, $(p, q, r)=(0.63,0.32,0.81)$ Blackwell dominates Option B, $(0.67,0.4,0.7){ }^{27}$ Subjects strictly preferring early resolution of uncertainty would be willing to pay for the former, while those strictly preferring

\footnotetext{
${ }^{26}$ These were framed and played out as lotteries in the Lottery treatment and as signals in the Information treatment, consistent with the rest of the experimental design.

${ }^{27}$ Due to a data recording error, we have responses to this question only from 7 individuals in the Information treatment and 59 individuals in the Lottery treatment.
} 
late resolution of uncertainty would be willing to pay for the latter. Finally, the third price list offers the choice between symmetric lotteries that differ only in skewness. Option A, $(0.91,0.45,0.98)$ is very positively skewed, while Option $\mathrm{B},(0.09,0.02,0.55)$ is equally informative but negatively skewed.

We find that $69.57 \%$ of subjects in the Lottery treatment and $68.49 \%$ of subjects in the Information treatment expressed a positive willingness to pay on at least one price list $(p=0.8906)$. This means that, for about $70 \%$ of subjects, we can conclude they are not simply indifferent among all possible lotteries or information structures. This represents a lower bound on strict preference-it's possible that subjects in the remaining $30 \%$ would be willing to pay for a lottery or information structure that was not represented as one of the 6 options in the price lists. Also interesting is the fact that there is no significant difference in willingness to pay across treatments. We see very different preferences over compound lotteries and information structures, but the same percentage of people have strict preference for information in these two environments.

\begin{tabular}{lccc}
\hline & Lotteries & Information & p-value \\
\hline One-Shot & & & \\
\hline One-Shot Early & $8.70 \%$ & $43.84 \%$ & 0.0000 \\
One-Shot Late & $31.88 \%$ & $6.85 \%$ & 0.0002 \\
p-value & 0.0025 & 0.0000 & \\
\hline Early/Late & & & \\
\hline Early & $11.86 \%$ & $57.14 \%$ & 0.0026 \\
Late & $35.59 \%$ & $0.00 \%$ & 0.058 \\
p-value & 0.0082 & - & \\
\hline Skewness & & & 0.27 \\
\hline Positive & $15.94 \%$ & $23.29 \%$ & 0.070 \\
Negative & $47.83 \%$ & $32.88 \%$ & \\
p-value & 0.0009 & 0.2743 & \\
\hline
\end{tabular}

Table VII: Percentage of subjects expressing positive willingness to pay in the price list elicitation questions

Table VII presents the percentage of subjects, across treatments, who were willing to pay for a particular type of resolution. The willingness-to-pay results confirm the main patterns from the choice data. In the Lottery treatment, subjects are significantly more likely to pay for late resolution than for early resolution, both when it's one-shot and when it's gradual. On the other hand, in the Information treatment, subjects are more likely to pay for early resolution than for late resolution ${ }^{28}$ Between treatment results hold, as wellmore subjects are willing to pay for early resolution in the Information treatment compared to the Lottery

\footnotetext{
${ }^{28}$ Note, around $30 \%$ of individuals are willing to pay for one-shot late resolution in the Lottery treatment, whereas only $17 \%$ chose one-shot late resolution in the unrestricted set. This suggests that there are some individuals who prefer gradual over one-shot resolution and chose a gradual lottery in the unrestricted set, but prefer one-shot late over one-shot early resolution in a binary comparison. For Information, on the other hand, roughly the same percentage of individuals are willing to pay for one-shot early as chose it in the unrestricted set.
} 
treatment, and are more likely to pay for late resolution in the Lottery treatment than in the Information treatment.

As a result, we conclude that our results are not an artifact of indifference. Subjects in the Lottery treatment express a strict preference for late resolution, while those in the Information treatment express a strict preference for early resolution. While information is entirely non-instrumental in both treatments, individuals are willing to pay to speed up or delay receipt of this information.

The general trend from the set choices is that individuals prefer negatively skewed lotteries and positivelyskewed information. The price list question confirms the former observation, as individuals in the Lottery treatment are significantly more willing to pay for the negatively skewed lottery than for the symmetric positively skewed lottery. However, we don't find that individuals in the Information treatment are significantly more likely to pay for positively skewed information. Recall that there existed a significant preference for positively skewed information only when we prevented one-shot late resolution of uncertainty. Both price list options, $(0.91,0.45,0.98)$ and $(0.09,0.02,0.55)$, are "close" to late resolution of uncertainty, where the subject is very likely to face a posterior near the prior. Our results suggest this is where skewness preferences over information structures are weakest.

\section{Discussion}

\section{Testing Theories}

Given the observed preferences, a natural question is which of the theories summarized in Table I], if any, capture observed preferences. The first observation is that preferences over information structures differ quite dramatically from preferences over information structure. No existing theory captures this difference. It would be very valuable to develop theoretical models that allow for preferences to vary with the time at which uncertainty resolves.

A primary feature of preferences over compound lotteries appears to be that individuals prefer intermediate resolution. Rather than choosing to resolve all uncertainty in the first or second stage, individuals choose gradual resolution. This rules out models like Kreps and Porteus (1978), Grant et al. (1998), and Kőszegi and Rabin (2009) which predict individuals will choose the earliest or latest resolution available. We find no evidence of a preference for one-shot resolution, further eliminating the theory of Dillenberger (2010). However, individuals are not indifferent over all forms of uncertainty resolution, as predicted in Segal (1990).

Ely et al. (2015) predicts a preference for gradual resolution, most similar to observed preferences. While we don't see choices exactly as predicted by the suspense and surprise models, these models come closest to capturing the observed preference for gradual resolution and a local preference for late resolution. The sus- 
pense and surprise models were designed to capture uncertainty such as sports games and mystery novels where the outcome hasn't occurred yet, which is indeed more consistent with our lottery domain. The preferences in Ely et al. (2015) do satisfy a strong symmetry assumption (Masatlioglu et al., 2017) and therefore should be indifferent to skewness, which is not the case in the lottery treatment. So while choices in the lottery treatment are best described by Ely et al. (2015), they do not perfectly fit all predictions of the models.

Over information structures, individuals prefer early resolution of uncertainty. This is consistent with the models of Kreps and Porteus (1978), Grant et al. (1998), and Köszegi and Rabin (2009). The only model consistent also with the observed preference for positive skew is that of Kreps and Porteus (1978). In their study of preferences over the resolution of uncertainty in an environment similar to our information treatment, Masatlioglu et al. (2017) also find preferences consistent with the model of Kreps and Porteus (1978).

\section{Qualitative Evidence}

In a non-incentivized questionnaire, we remind subjects of their choice in the unrestricted set and ask them to explain why they chose that particular lottery or information structure. Very few subjects mentioned choosing randomly. Responses indicate that subjects understood they could not affect the overall odds, but subjects in the Information treatment are very clear in preferring early information for the benefit of knowing the lottery outcome sooner. That is, the strong preference for early resolution that we see in the data is exactly what subjects express qualitatively. A few representative quotes, transcribed directly, can be found below.

- "I do not like surprises and I get very anxious when I do not know something for certain, so I wanted the signal to tell me exactly what was going to happen. I wanted the signal to tell me exactly what was going to happen so I did not have to wait."

- "I opted to make sure I knew what prize I was getting based on my signal."

- "I figured why not try to know if I got the high prize or not becasue there was no penatly to not knowing. If I can be sure without a cost, I guess I prefer that. Less wondering and you can move on."

- "I did not pay much attention to the odds of recieving a red or blue signal, I focused mostly on being sure that if I saw a red signal, I'd be very certian I won a high payment, and if I saw a blue signal, I'd be very certain I'd recieved the low prize."

Recall that we did not find much evidence for one-shot resolution of uncertainty. However, one subject's responses perfectly captured the preference -

"if $i$ had the option of knowing $100 \%$ for something $i$ went with that, other wise i went with 50/50 because i'd rather know for sure or not at all. i dont want to get my hopes up just to lose."

In the Lottery treatment, it's clear that some some subjects viewed all lotteries identically, and the experiment setup induced reduction of compound lotteries-“There wasn't much thought since all the chances were 
50/50." Subjects exhibiting preference for early resolution of uncertainty were clear in their motivation for their choices. Subjects choosing for uncertainty to resolve in Stage 1 of the lottery did so in order to learn the lottery outcome sooner and not based on other factors irrelevant to our motivation.

- "Made Urn 2 as close to $100 \%$ red as possible so I would know after Task 1 if I got $\$ 11$ or $2 . "$

- "As much as possible I wanted my payout to be determined by urn choice so I wouldn't have to wait to know if I had won or lost."

- "Chose the set of probabilities that led to having all of the balls in either Urn 1 or Urn 2. I did this so I would know right away what my profitability would be."

Interestingly, subjects exhibiting a preference for late resolution of uncertainty give two types of motivations. The first is the expected preference to avoid information, or to design uncertainty such that it resolves later. Subjects' responses allude to avoiding disappointment in finding out they did not win the high prize- 'I didn't want anything to be for sure blue until the end since I wanted the largest payout." Some responses even seem to indicate a preference for one-shot late resolution 29

- "I usually liked just one urn to be at 100\% then for that urn to be a 50:50 chance. I know it was all a 50\% chance regardless, but I liked my luck being based on only 1 event versus two."

- "I like the simplicity of this lottery. I have one 50/50 chance at getting red."

On the other hand, many subjects stated a preference for "diversification," which manifests as a preference for late resolution. These subjects try to have a "high enough" posterior regardless of which urn is selected in Stage 1. Often this results in equalizing odds across all states of the world, or at least reducing the variance in posteriors in the second stage of the lottery.

- "I figured it didn't matter much since the probability would be 50/50 either way, but I tried to keep each urn as close to $50 \%$ because I knew I would feel better if, no matter which lottery was selected and no matter which urn from that lottery was selected, there was still a reasonable chance of getting a red ball."

- "I wanted to have a larger probability of selecting Urn 2, which had a majority of red balls. However, still wanted to have a chance if Urn 1 was selected"

- "I was banking on urn 2 getting chosen and a red ball being chosen from urn 2 . However, urn 1 still had some chance of getting the high prize if chosen."

These subjects are averse to learning the lottery outcome in the first stage, but not quite because they are simply avoiding information. They avoid resolution in the first stage because they don't want to face a state of the world, or an urn, in this case, in which they know they have no chance of winning. They feel as if they haven't lost yet, and don't want to expedite the possibility of guaranteeing a loss.

\footnotetext{
${ }^{29}$ In a hypothetical question between Stage 1 and Stage 2 realization, we asked subjects to rate, on a scale from 1-7, their disappointment after seeing Stage 1 realization. Subjects in the Information treatment report significantly higher disappointment levels (4.07 vs. $3.19, p=0.030$ ). This makes sense since subjects in the Information treatment resolve more uncertainty earlier, and had a higher potential to be disappointed.
} 
We hypothesize that these other factors may contribute to the difference between the treatments. In the Information treatment, the outcome has already been determined and subjects simply want to know the result. When the lottery outcome has not been determined, other psychological factors such as luck, illusion of control, etc. may come into play (Langer, 1975; Eliaz and Frechette, 2013). Winning the high or low prize is not yet set in stone, and subjects are averse to locking in the low prize earlier.

In a hypothetical question between Stage 1 and Stage 2 resolution, we present subjects with the environment from Eliaz and Frechette (2013), which measures a form of "false diversification." We compare the false diversification tendency between those who choose earliest resolution in Set 1 and those who choose to delay some uncertainty resolution. We find that subjects in the Lottery treatment who do not choose early resolution of uncertainty are significantly more likely to prefer this false diversification $(62 \%$ vs. $22 \%, p=0.026)$. In the Information treatment, however, there is no significant difference (68\% vs. $73 \%, p=0.63)$. This gives suggestive evidence that individuals in the Lottery treatment delayed resolution for false diversification and other psychological reasons stemming from the fact that uncertainty had not been resolved yet, while individuals in the Information treatment do not. Better understanding this difference is an interesting question for future research.

\section{CONCLUSION}

We demonstrate experimentally that preferences over information structures are not equivalent to preferences over compound lotteries in environments with resolving uncertainty. Instead, preferences for receiving information appear to follow the time at which the outcome occurs. In compound lotteries, when the outcome is determined in the last stage, individuals prefer to delay uncertainty resolution and learn information later. In information structures, when the outcome has already been determined, individuals prefer to expedite uncertainty resolution and learn information sooner. In both scenarios, we see a strong tendency for individuals to choose an intermediate form of uncertainty resolution. Testing recent theories in the literature, we find no evidence for a pervasive preference for one-shot resolution of uncertainty. Individuals prefer one-shot early resolution of information structures and one-shot late resolution of lotteries, but the two types of resolution are not substitutes, as Dillenberger (2010) assumes, nor are they both preferred to any gradual resolution as Dillenberger (2010) and Köszegi and Rabin (2009) predict.

As a result, we conclude that information avoidance will be more prevalent about outcomes which will come to fruition in the future. On the other hand, individuals will pay to learn outcomes which have been resolved, even when this information is not instrumental. This can help us classify field environments and predict situations in which individuals will require extra incentives to acquire information. Policy makers could 
reframe these environments as information structures, focusing on aspects of the outcome which have already occurred which would allow them to take advantage of individuals' natural tendency to acquire information in these environments.

Our experimental results leave open a number of interesting questions for future research. Of primary importance for theory is the result that preferences over lotteries are not the same as preferences over information structures. Descriptive theories in this domain would need to allow for preferences to vary with the time at which uncertainty resolves.

Additionally, our experiment studies positive outcomes of a lottery. While none of the existing theories in the literature predict preferences to differ between positive and negative outcomes, it would be interesting to compare the two domains. Behavioral biases could lead to different preferences over negative outcomes. Our experiment also uses a fairly limited 30 minute time delay. It would be very interesting to find a way to study preferences over a long horizon, such as days or years, while still keeping information non-instrumental. Furthermore, previous studies (Ganguly and Tasoff, 2016, Masatlioglu et al., 2017) have found information preferences can change with the stakes and with the prior. Future research can better understand how varying stakes and priors interact with the difference between lotteries and information structures.

Finally, it's unclear to what extent these preferences would carry over into domains where early resolution of uncertainty could be instrumental. This introduces many other factors which may interact differently between lotteries and information structures. It would be valuable for experiments to be able to disentangle instrumental from non-instrumental considerations in information acquisition to identify what portion of information acquisition or avoidance is driven by underlying information preferences. 


\section{REFERENCES}

Ahlbrecht, M., Weber, M., 1997. Preference for gradual resolution of uncertainty. Theory and Decision 43, $167-185$.

Allais, M., 1953. The foundations of a positive theory of choice involving risk and a criticism of the postulates and axioms of the american school. Econometrica 21, 503-546.

Andreoni, J., Sprenger, C., 2012a. Estimating time preferences from convex budgets. American Economic Review 102 (7), 3333-3356.

Andreoni, J., Sprenger, C., 2012b. Risk preferences are not time prefereces. American Economic Review 102 (7), $3357-3376$.

Aria, D., 1997. Temporal resolution of uncertainty in risky choices. Acta Psychologica 96, 15-26.

Azrieli, Y., Chambers, C. P., Healy, P. J., forthcoming. Incentives in experiments: A theoretical analysis. Journal of Political Economy.

Boiney, L. G., 1993. The effects of skewed probability on decision making under ambiguity. Organizational Behavior and Human Decision Processes 56, 134-148.

Brown, A., Kim, H., 2013. Do individuals have preferences used in macro-finance models? an experimental investigation. Management Science 60 (4), 939-958.

Burling, S., 2017. Testing for alzheimer's genes forces difficult decisions. URL http: //www . chicagotribune.com/news/nationworld/sns-tns-bc-med-genetictesting-20171221-story . html

Chew, S., Ho, J., 1994. Hope: An empirical study of attitude toward the timing of uncertainty resolution. Journal of Risk and Uncertainty 8 (3), 267-288.

Dean, M., Ortoleva, P., 2016. Is it all connected? a testing ground for unified theories of behavioral economics phenomena. Working Paper.

Dillenberger, D., November 2010. Preferences for one-shot resolution of uncertainty and allais-type behavior. Econometrica 78 (6), 1973-2004.

Eliaz, K., Frechette, G., 2013. “don't put all your eggs in one basket!”: An experimental study of false diversification. Working Paper. 
Eliaz, K., Schotter, A., 2007. Experimental testing of intrinsic preferences for non-instrumental information. American Economic Review (Papers and Proceedings) 97, 166-169.

Eliaz, K., Schotter, A., 2010. Paying for confidence: An experimental study of the demand for non-instrumental information. Games and Economic Behavior.

Ely, J., Frankel, A., Kamenica, E., 2015. Suspense and surprise. Journal of Political Economy 123 (1), $215-260$.

Epstein, L. G., Zin, S. E., 1989. Substitution, risk aversion, and the temporal behavior of consumption and asset returns: A theoretical framework. Econometrica, 937-969.

Falk, A., Zimmermann, F., 2016. Beliefs and utility: Experimental evidence on preferences for information. Working Paper.

Fischbacher, U., 2007. z-tree: Zurich toolbox for ready-made economic experiments. Experimental Economics $10(2)$.

Ganguly, A. R., Tasoff, J., 2016. Fantasy and dread: the demand for information and the consumption utility of the future. Management Science 63 (12), 4037-4060.

Golman, R., Hagmann, D., Loewenstein, G., 2016. Information avoidance. Journal of Economic Literature.

Grant, S., Kajii, A., Polak, B., 1998. Intrinsic preference for information. Journal of Economic Theory 83, 233-259.

Greiner, B., 2004. An online recruitment system for economics experiments.

Gul, F., 1991. A theory of disappointment aversion. Econometrica 59 (3), 667-686.

Kahneman, D., Tversky, A., March 1979. Prospect theory: An analysis of decision under risk. Econometrica $47(2), 263-292$.

Kőszegi, B., Rabin, M., 2009. Reference-dependent consumption plans. American Economic Review 99 (3), 909-936.

Kocher, M., Krawczyk, M., van Winden, F., 2014. Let me dream on! anticipatory emotions and preferences for timing in lotteries. Journal of Economic Behavior and Organization 98, 29-40.

Kreps, D. M., Porteus, E. L., 1978. Temporal resolution of uncertainty and dynamic choice theory. Econometrica, $185-200$.

Langer, E. J., 1975. The illusion of control. Journal of Personality and Social Psychology 32, 311-328. 
Loewenstein, G., 1987. Antcipation and the valuation of delayed consumption. The Economic Journal, 666684.

Lovallo, D., Kahneman, D., 2000. Living with uncertainty: Attractiveness and resolution timing. Journal of Behavioral Decision Making 13 (2).

Masatlioglu, Y., Orhun, A. Y., Raymond, C., 2017. Intrinsic information preferences and skewness. Working Paper.

Meissner, Thomasand Pfeiffer, P., 2017. Measuring preferences over the temporal resolution of consumption uncertainty. Working Paper.

Palacios-Huerta, I., 1999. The aversion to the sequential resolution of uncertainty. Journal of Risk and Uncertainty 18, 249-269.

Poulsen, A. U., Roos, M. W. M., 2010. Do people make strategic commitments? experimental evidence on strategic information avoidance. Experimental Economics 13, 206-225.

Rothbart, M., Snyder, M., 1970. Confidence in the prediction and postdiction of an uncertain outcome. Canadian Journal of Behavioural Science/Revue canadienne des sciences du comportement 2, 38.

Segal, U., 1990. Two-stage lotteries without the reduction axiom. Econometrica 58 (2), 349-377.

Von Gaudecker, H.-M., Van Soest, A., Wengsträm, E., 2011. Heterogeneity in risky choice behaior in a broad population. The American Economic Review, 664-694.

Zimmermann, F., 2015. Clumped or piecewise? - evidence on preferences for information. Management Science $61(4), 740-753$. 


\section{A. Choice Data in Each Set}

The graphs below plot all subjects' choices in $(p, q, r)$ space, separated by treatment. One-shot early, one-shot late, earliest, and latest possible resolution are labeled. Plot points are weighted by frequency of choice with bigger circles indicating that a larger number of subjects chose a particular lottery or information structure.

Set 1

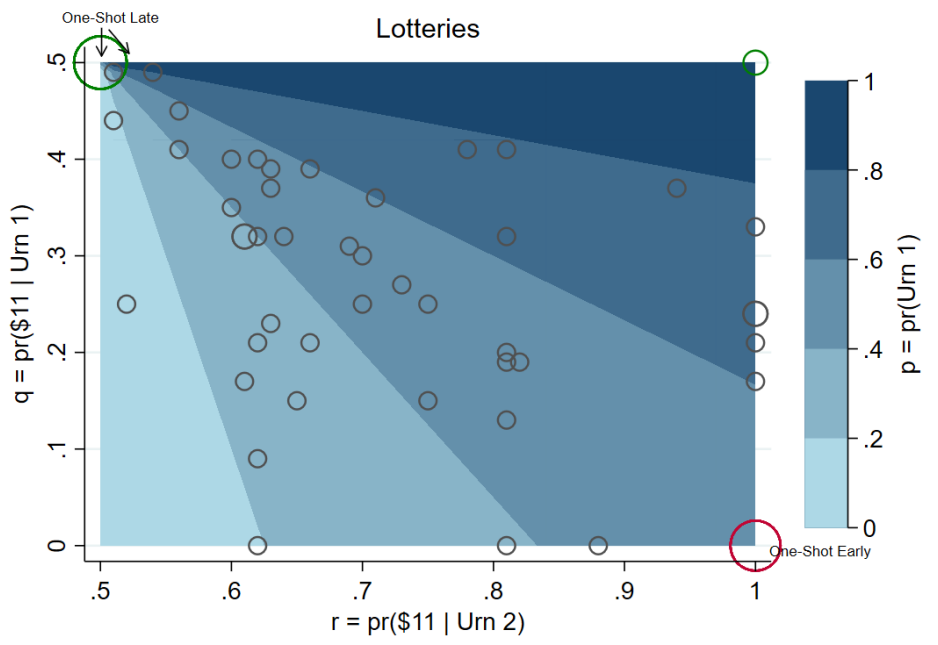

(1) Lottery Treatment
Set 1

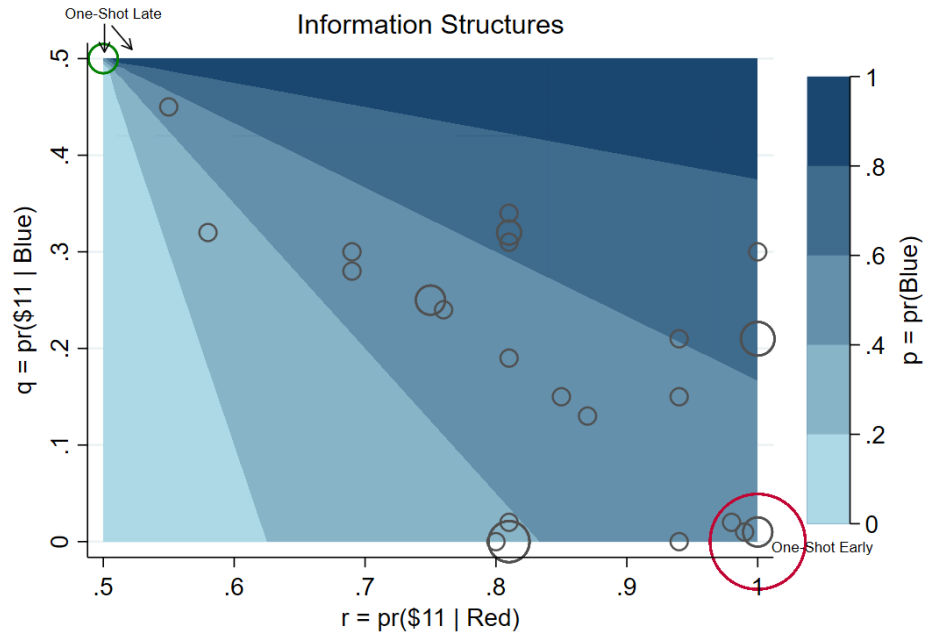

(2) Information Treatment

Figure VI: Unrestricted set choices

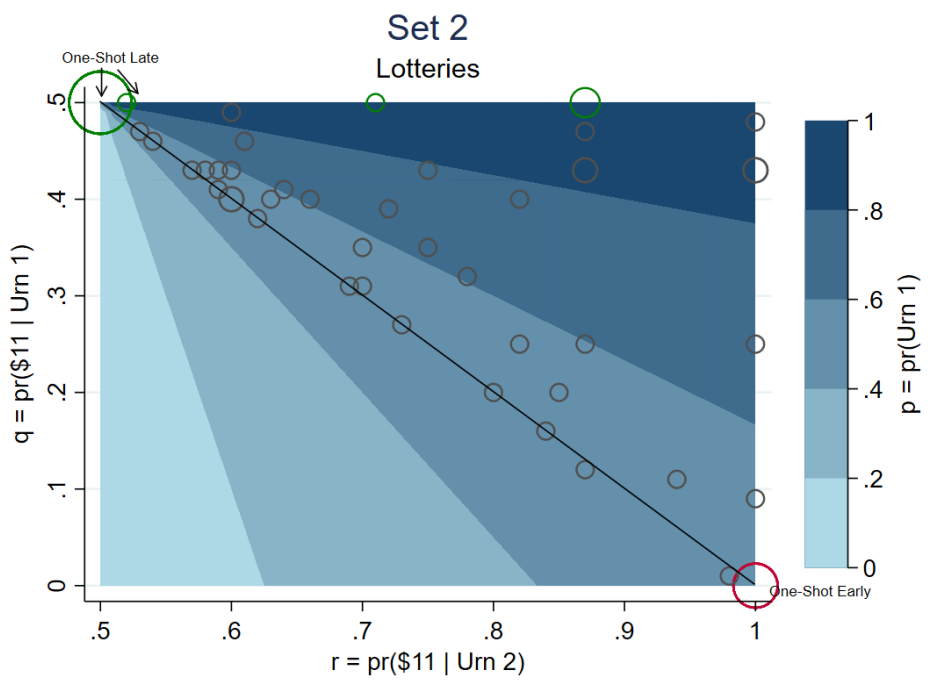

(1) Lottery Treatment

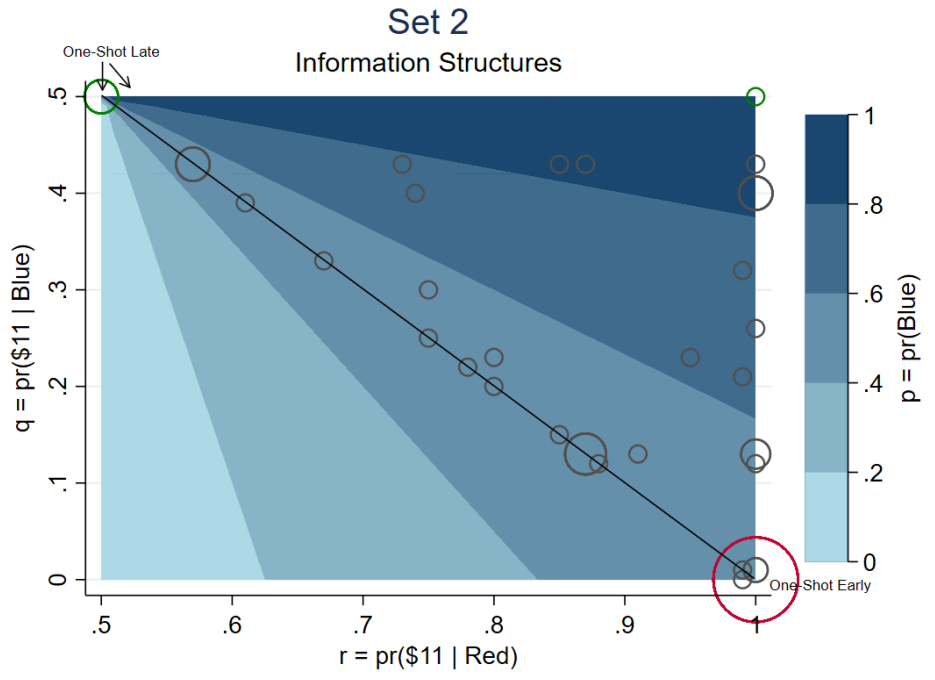

(2) Information Treatment

Figure VII: Only positively skewed 
Set 3

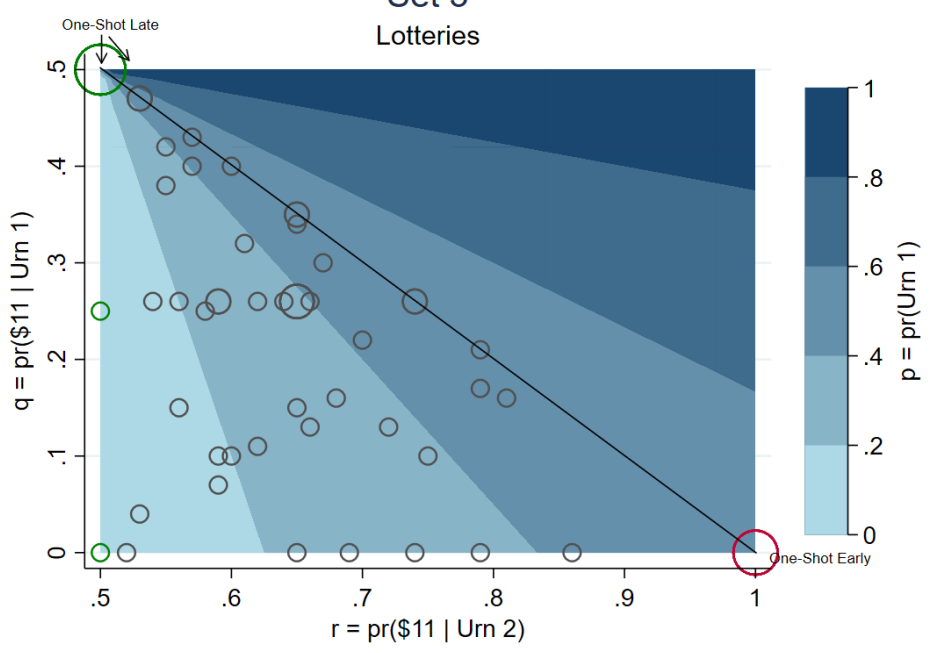

(1) Lottery Treatment
Set 3

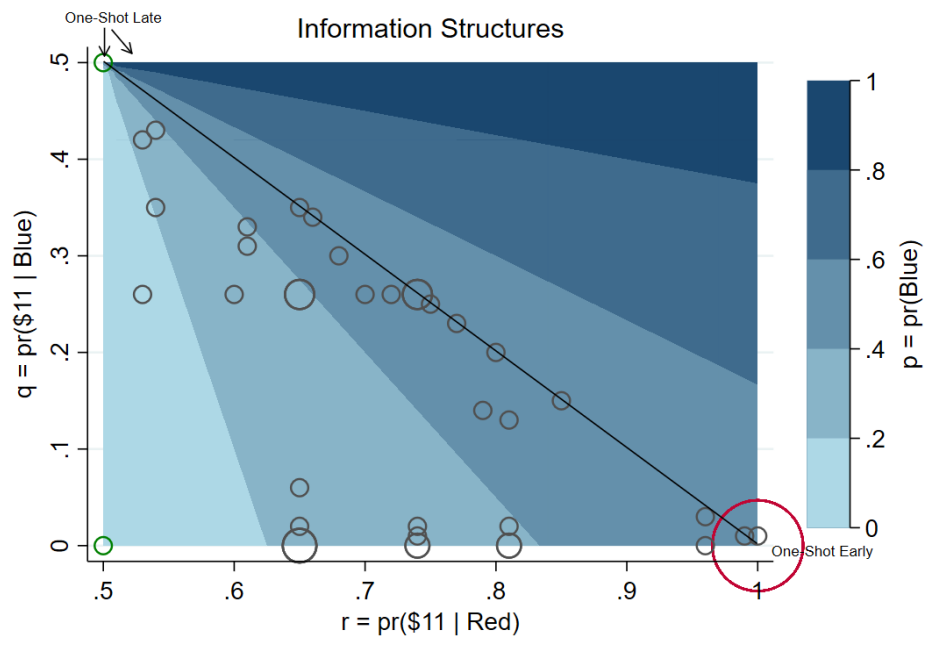

(2) Information Treatment

Figure VIII: Only negatively skewed

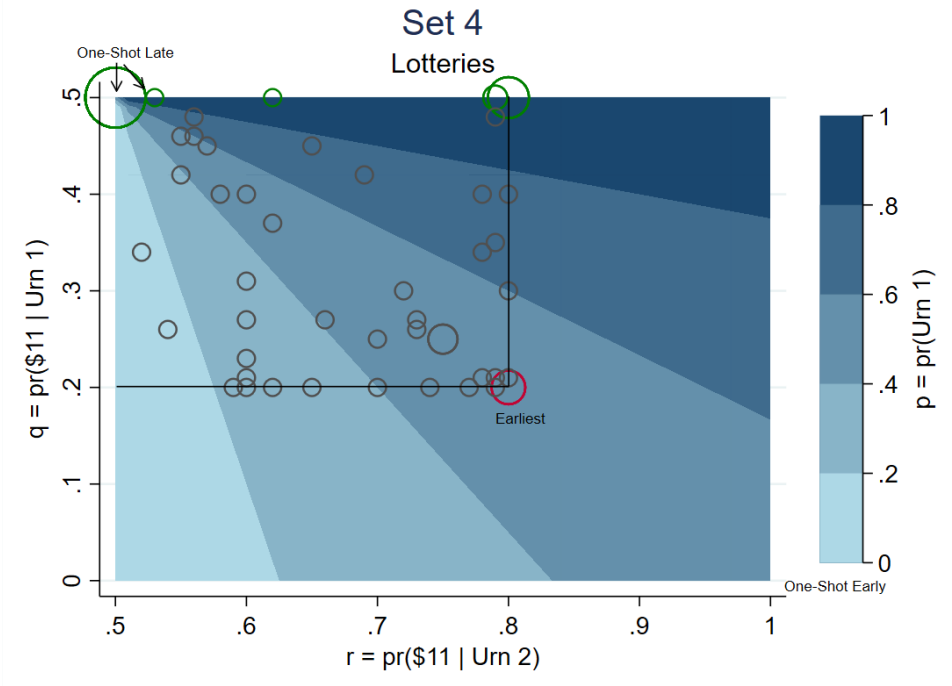

(1) Lottery Treatment

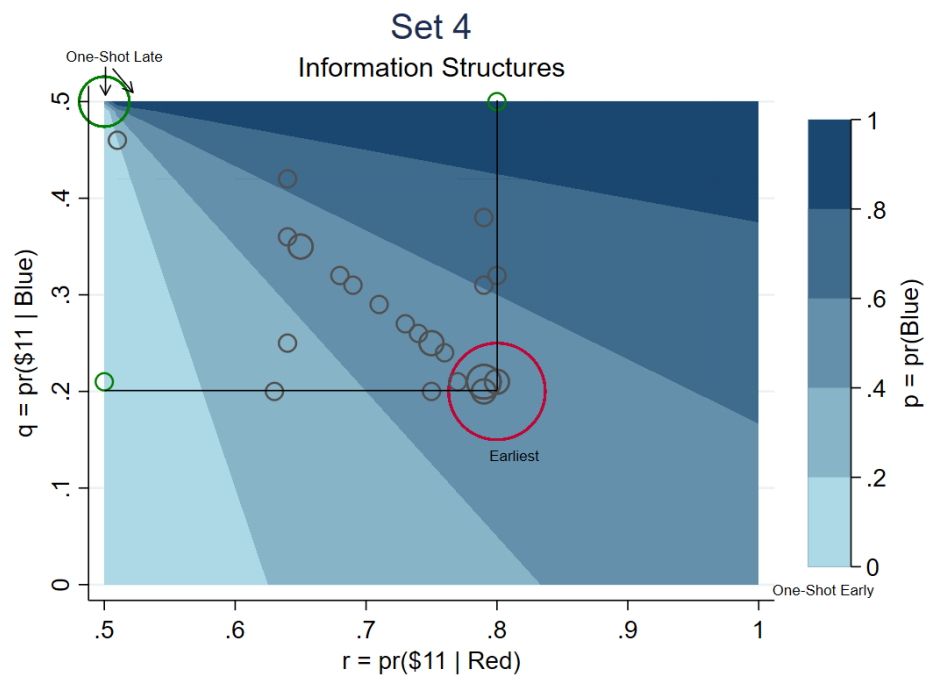

(2) Information Treatment

Figure IX: Prevent One-Shot Early Resolution 


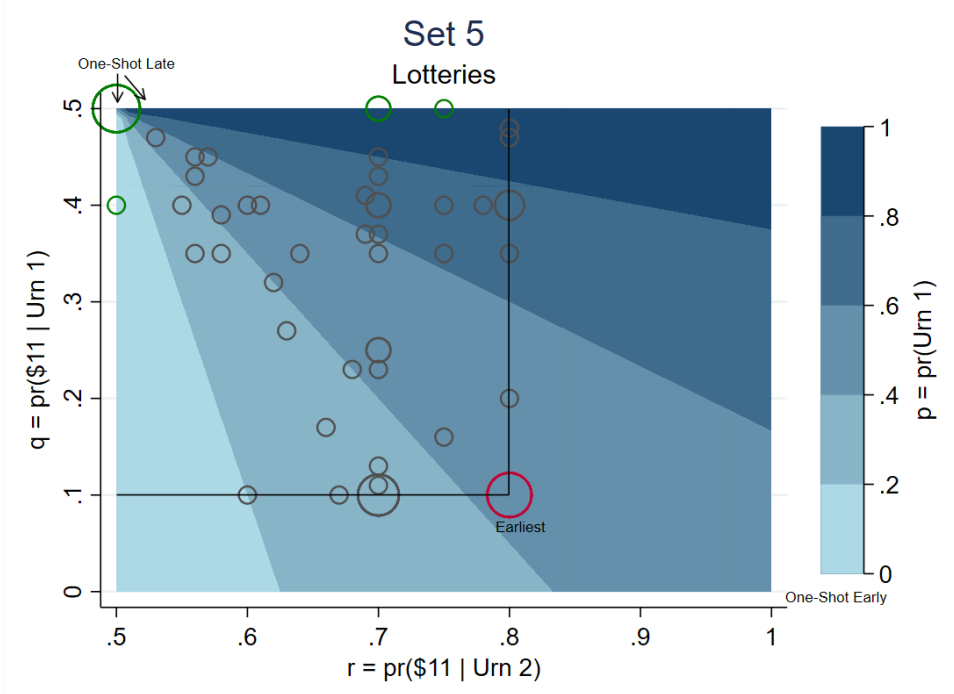

(1) Lottery Treatment

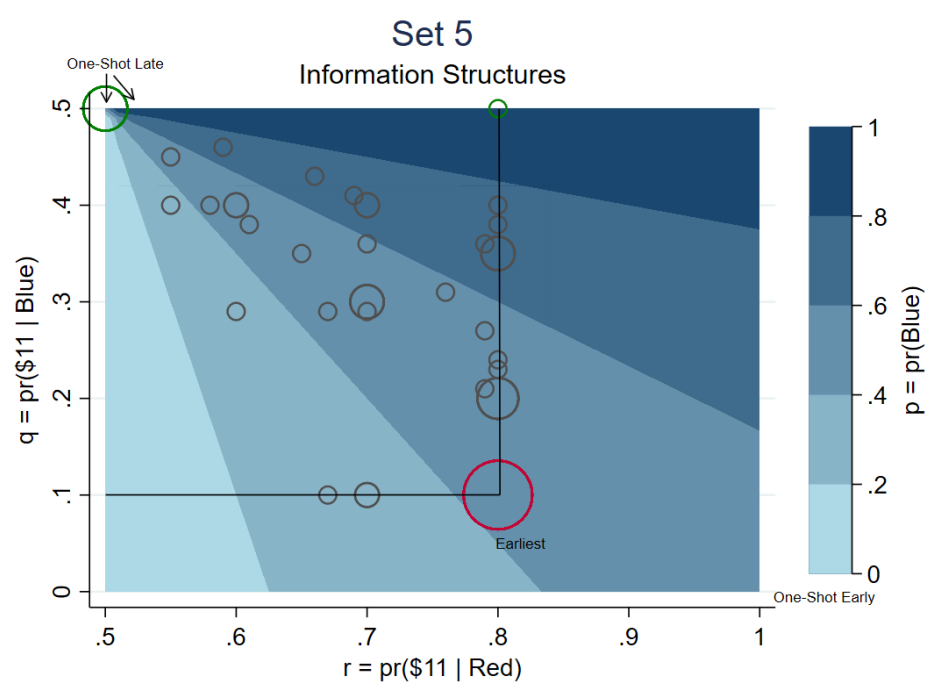

(2) Information Treatment

Figure X: Prevent One-Shot Early Resolution

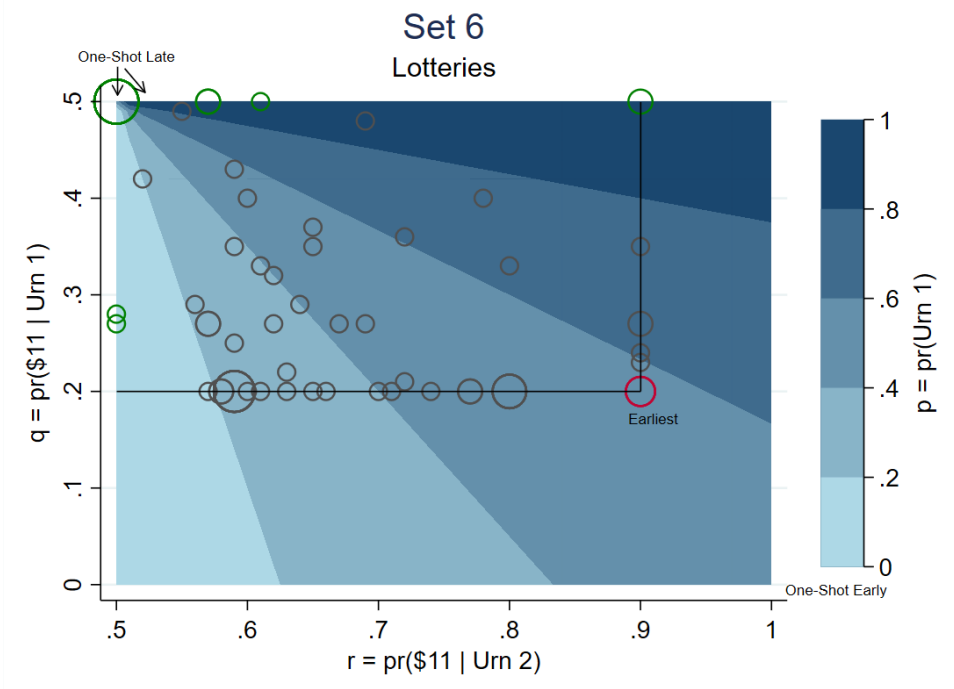

(1) Lottery Treatment

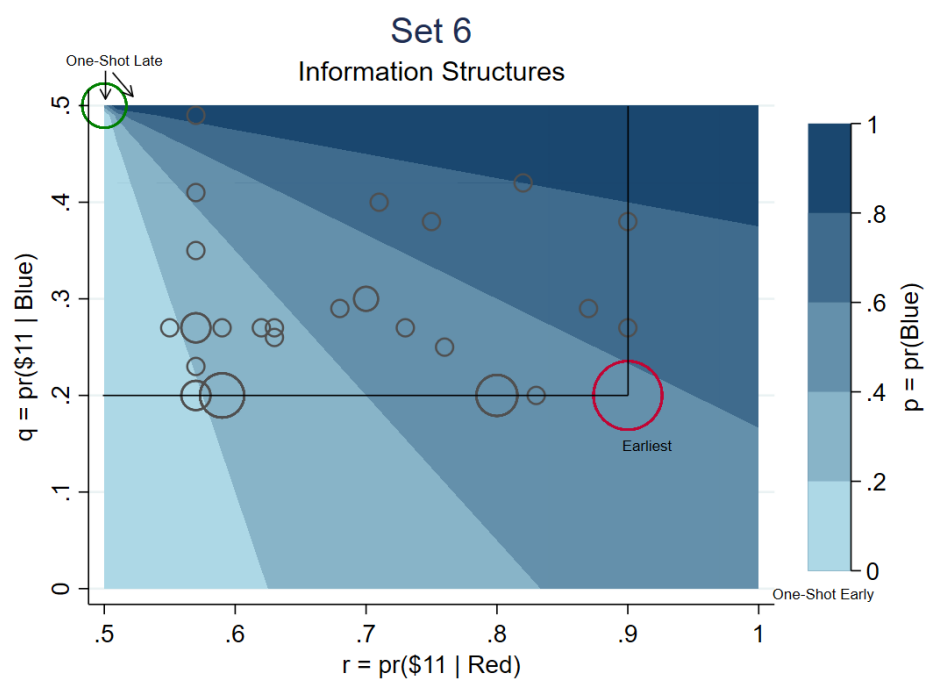

(2) Information Treatment

Figure XI: Prevent One-Shot Early Resolution 


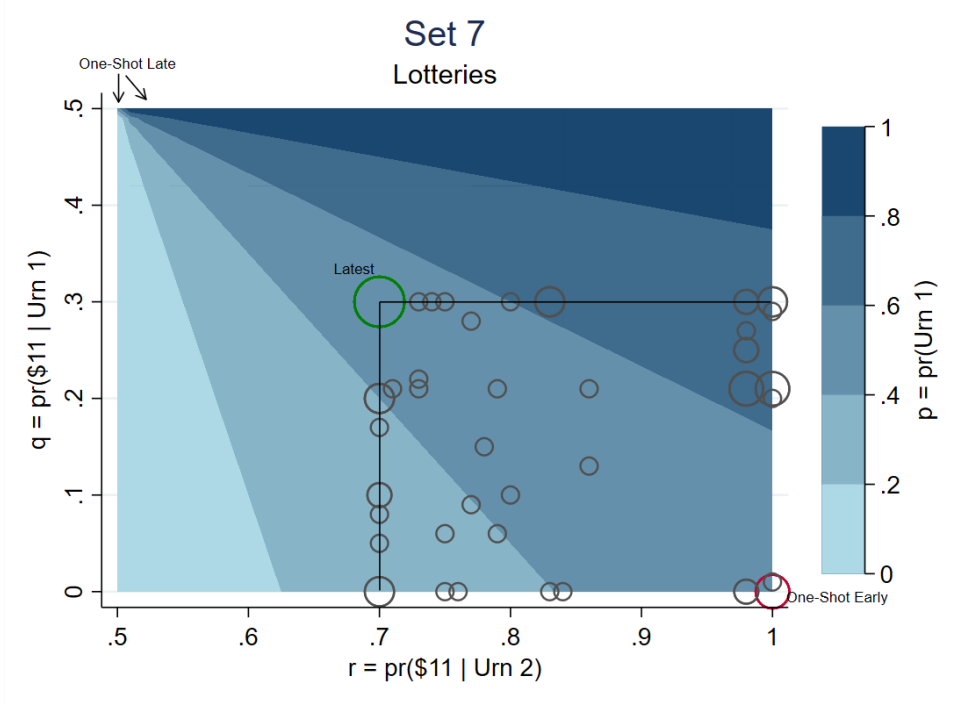

(1) Lottery Treatment

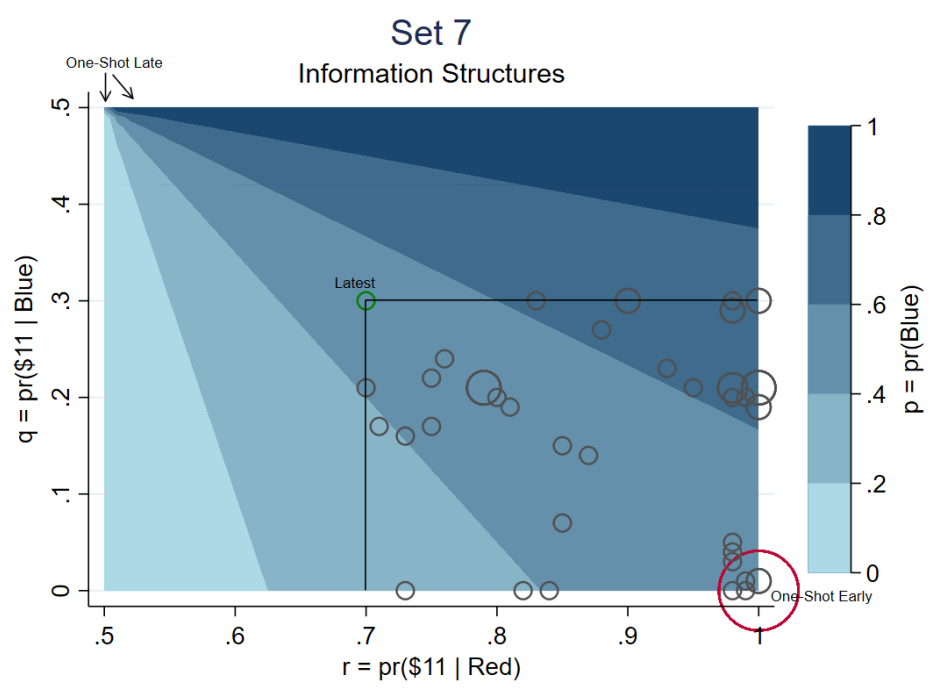

(2) Information Treatment

Figure XII: Prevent One-Shot Late Resolution

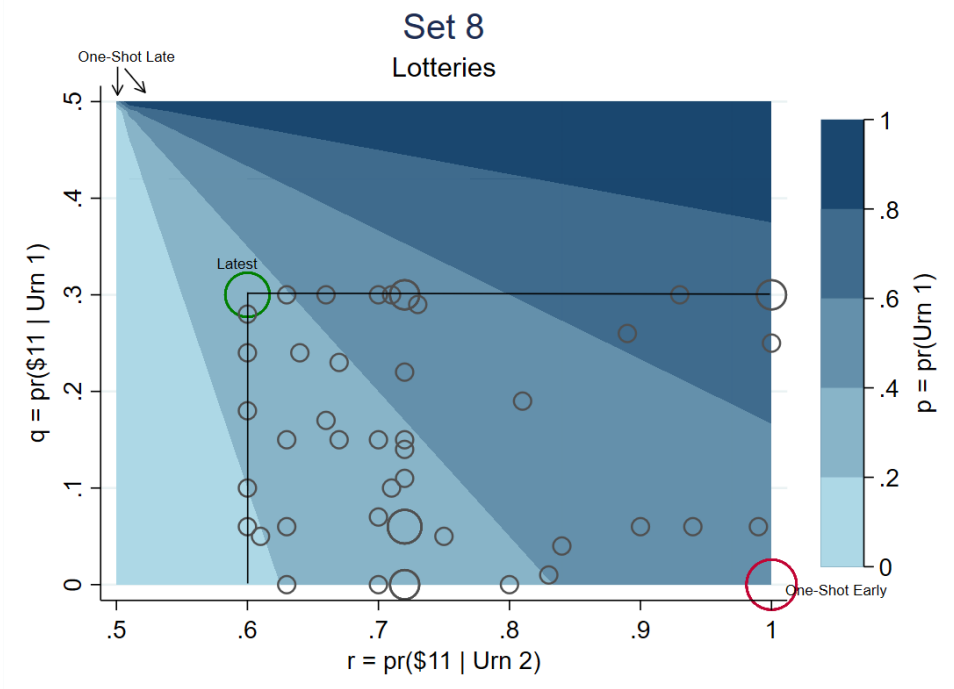

(1) Lottery Treatment

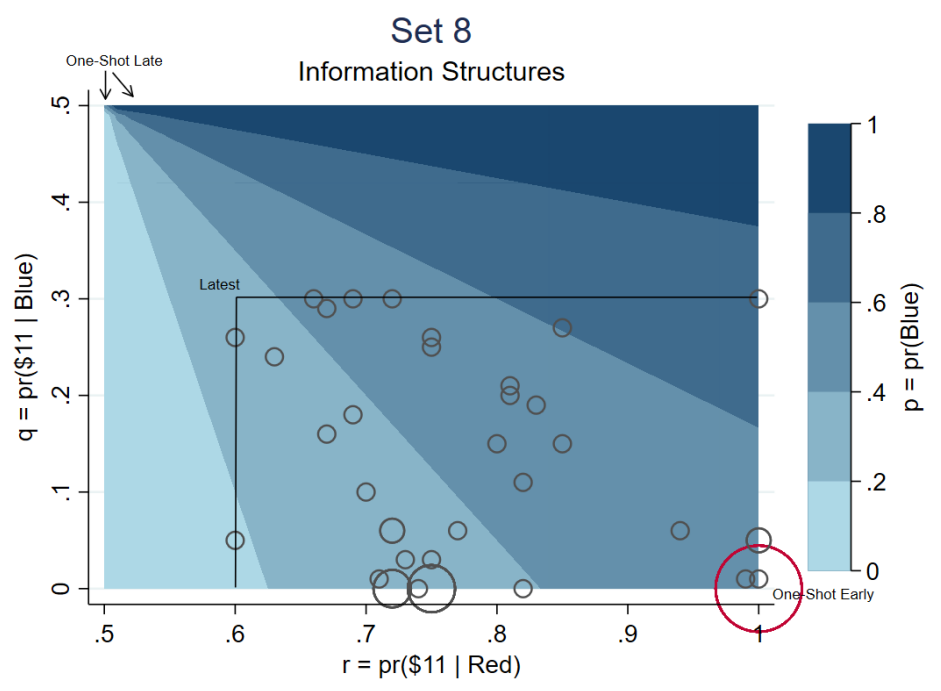

(2) Information Treatment

Figure XIII: Prevent One-Shot Late Resolution 


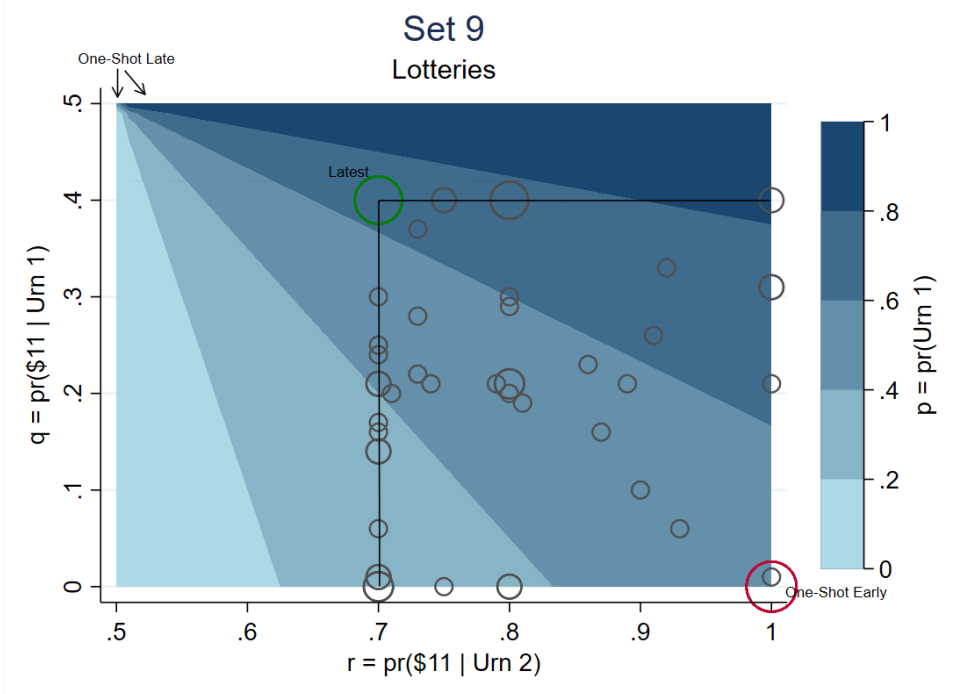

(1) Lottery Treatment

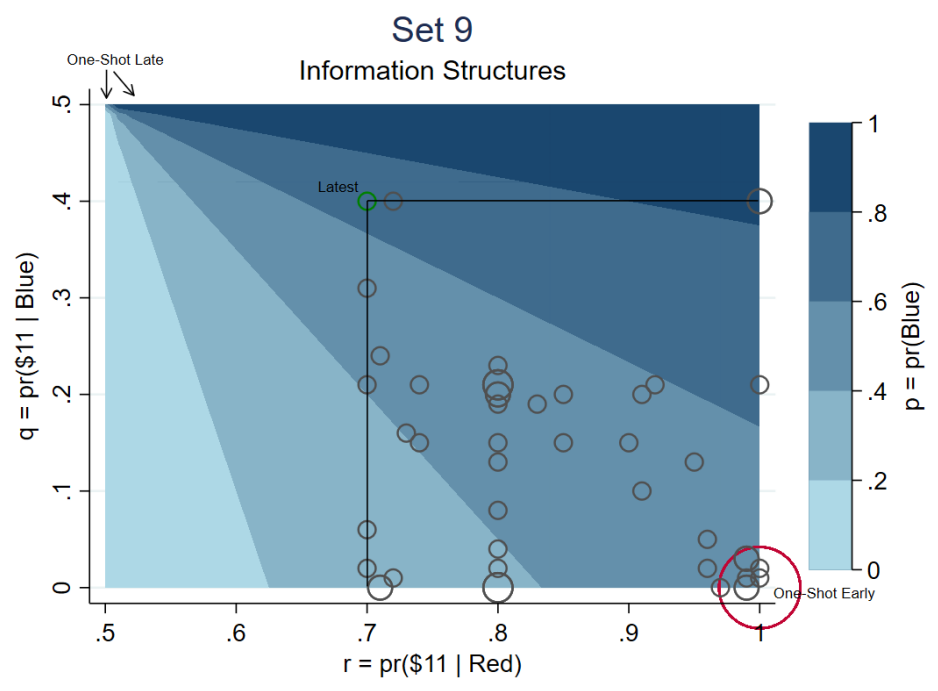

(2) Information Treatment

Figure XIV: Prevent One-Shot Late Resolution

\section{B. Additional Results}

\begin{tabular}{ccccccc}
\hline \multicolumn{3}{c}{ Set } & \multicolumn{3}{c}{ Percentage of Early Choices } & \multicolumn{3}{c}{ Percentage of Late Choices } \\
Lotteries & Information & p-value & Lotteries & Information & p-value \\
\hline 1 & $13.04^{\dagger}$ & $45.21^{\dagger}$ & .0000 & $17.39^{\dagger}$ & $4.11^{\dagger}$ & .0103 \\
2 & $10.14^{\dagger}$ & $35.62^{\dagger}$ & .0003 & $30.43^{\dagger}$ & $6.85^{\dagger}$ & .0003 \\
3 & $10.14^{\dagger}$ & $41.10^{\dagger}$ & .0000 & $20.29^{\dagger}$ & $2.74^{\dagger}$ & .0010 \\
\hline \multicolumn{2}{l}{ Prevent } & One-Shot Early Resolution: & & & & \\
\hline 4 & 5.80 & 46.58 & .0000 & $34.78^{\dagger}$ & $15.07^{\dagger}$ & .0066 \\
5 & 10.14 & 31.51 & .0019 & $20.29^{\dagger}$ & $10.96^{\dagger}$ & .1259 \\
6 & 4.35 & 32.88 & .0000 & $20.29^{\dagger}$ & $9.59^{\dagger}$ & .0736 \\
\hline \multicolumn{2}{l}{ Prevent One-Shot Late Resolution: } & & & & \\
\hline 7 & $5.80^{\dagger}$ & $31.51^{\dagger}$ & .0001 & 13.04 & 1.37 & .0068 \\
8 & $13.04^{\dagger}$ & $36.99^{\dagger}$ & .0011 & 10.14 & 0.00 & .0054 \\
9 & $13.04^{\dagger}$ & $32.88^{\dagger}$ & .0053 & 11.59 & 1.37 & .0128 \\
\hline
\end{tabular}

Table VIII: Percentage of Early and Late Choices in Each Set, by treatment. Values with a dagger indicate one-shot resolution. Reported p-values are from two-tailed Mann-Whitney ranksum tests. All significance levels also hold with ranksum tests of equality on the medians. 


\begin{tabular}{|c|c|c|c|}
\hline Set & Lotteries & Information & p-value \\
\hline 1 & 30.43 & 49.32 & .0223 \\
\hline 2 & 40.58 & 42.47 & .8203 \\
\hline 3 & 30.43 & 43.84 & .1001 \\
\hline \multicolumn{4}{|c|}{ Prevent One-Shot Early Resolution: } \\
\hline 4 & 34.78 & 15.07 & .0066 \\
\hline 5 & 20.29 & 10.96 & .1259 \\
\hline 6 & 20.29 & 9.59 & .0736 \\
\hline \multicolumn{4}{|c|}{ Prevent One-Shot Late Resolution: } \\
\hline 7 & 5.80 & 31.51 & .0001 \\
\hline 8 & 13.04 & 36.99 & .0011 \\
\hline 9 & 13.04 & 32.88 & .0053 \\
\hline
\end{tabular}

Table IX: Percentage of One-Shot Choices in Each Set

C. Transitions

\begin{tabular}{lcccc}
\hline & Earliest & Latest & Other & \\
\hline Set 4 & & & & \\
\hline Lotteries & $44.44 \%$ & $22.22 \%$ & $33.33 \%$ & $\mathrm{n}=9$ \\
Information & $72.73 \%$ & $9.09 \%$ & $18.18 \%$ & $\mathrm{n}=33$ \\
\hline Set 5 & & & & \\
\hline Lotteries & $33.33 \%$ & $11.11 \%$ & $55.55 \%$ & $\mathrm{n}=9$ \\
Information & $57.58 \%$ & $12.12 \%$ & $30.30 \%$ & $\mathrm{n}=33$ \\
\hline Set 6 & & & & \\
\hline Lotteries & $33.33 \%$ & $11.11 \%$ & $55.55 \%$ & $\mathrm{n}=9$ \\
Information & $63.64 \%$ & $12.12 \%$ & $24.24 \%$ & $\mathrm{n}=33$ \\
\hline
\end{tabular}

Table X: Choices when one-shot early is prevented, among those who choose one-shot early resolution in the unrestricted set

\begin{tabular}{lcccc}
\hline & Earliest & Latest & Other & \\
\hline Set 7 & & & & \\
\hline Lotteries & $0.00 \%$ & $25 \%$ & $75 \%$ & $\mathrm{n}=12$ \\
Information & $0.00 \%$ & $0.00 \%$ & $100 \%$ & $\mathrm{n}=3$ \\
\hline Set 8 & & & & \\
\hline Lotteries & $8.33 \%$ & $25 \%$ & $66.67 \%$ & $\mathrm{n}=12$ \\
Information & $0.00 \%$ & $0.00 \%$ & $100 \%$ & $\mathrm{n}=3$ \\
\hline Set 9 & & & & \\
\hline Lotteries & $8.33 \%$ & $16.67 \%$ & $75 \%$ & $\mathrm{n}=12$ \\
Information & $0.00 \%$ & $0.00 \%$ & $100 \%$ & $\mathrm{n}=3$ \\
\hline
\end{tabular}

Table XI: Choices when one-shot late is prevented, among those who choose one-shot late resolution in the unrestricted set 


\section{Determinants of Preferences}

Recall that, in Part 2 of the experiment, we elicited various measures of risk and time preferences following procedures in Dean and Ortoleva (2016). The elicited characteristics and their measurements are summarized in Table XII. We look to see whether choices of early, late, and one-shot resolution are correlated with individual measures of risk and time preferences.

\begin{tabular}{ll}
\hline Characteristic & Measurement \\
\hline Discount Rate & Value $x$ in 5 weeks of $\$ 6$ in 6 weeks as a percentage of 6 \\
Discount Rate (Present) & Value $y$ today of $\$ 6$ in 1 week as a percentage of 6 \\
Present Bias & $(x-y) / 6$ \\
Risk Aversion & 3 minus the certainty equivalent of lottery $(\$ 6,0.5 ; \$ 0)$ as a percent- \\
& age of 3 \\
Common Consequence & Difference between $z$, such that $\$ 4$ for sure is indifferent to \\
& $(\$ 4,0.89 ; \$ x, 0.1 ; \$ 0)$, and $y$, such that $(\$ 4,0.11 ; \$ 0)$ is indifferent to \\
& $(\$ y, 0.10 ; \$ 0)$ as a percentage of 4 \\
Common Ratio & Difference between $z$, such that $\$ 4$ for sure is indifferent \\
& to $(\$ x, 0.8 ; \$ 0)$ and $y$, such that $(\$ 4,0.25 ; \$ 0)$ is indifferent to \\
& $(\$ y, 0.2 ; \$ 0)$ as a percentage of 4 \\
\hline
\end{tabular}

Table XII: Risk and Time Preference Elicitation

Table XIII reports correlations between willingness to pay in the price list questions and various measures of risk and time preferences. The first rows report willingness to pay in the Lottery treatment, while the last rows report willingness to pay in the Information treatment. The general result from the Lottery treatment is that more patient individuals, with higher value for delta, are less likely to pay for early resolution, both one-shot early and when early resolution is gradual. The same holds in the Information treatment, but much less strongly. Presumably, this is because a preference for early resolution is more universal in the Information treatment and is less determined by individual preferences. 


\begin{tabular}{|c|c|c|c|c|}
\hline & Discount Rate & Discount Rate (Present) & Present Bias & Risk Aversion \\
\hline \multicolumn{5}{|c|}{ LOTTERIES } \\
\hline \multicolumn{5}{|c|}{ OS Early vs. OS Late } \\
\hline OS Early & $-0.4037 * * *$ & $-0.2230^{*}$ & -0.0770 & $0.2183^{*}$ \\
\hline OS Late & -0.0951 & -0.0437 & -0.0262 & -0.1676 \\
\hline \multicolumn{5}{|c|}{ Early vs. Late } \\
\hline Early & $-0.5269 * * *$ & $-0.2613^{* *}$ & -0.1736 & $0.2964^{* *}$ \\
\hline Late & 0.1331 & -0.1159 & 0.2158 & -0.1531 \\
\hline \multicolumn{5}{|c|}{ INFORMATION } \\
\hline \multicolumn{5}{|c|}{ OS Early vs. OS Late } \\
\hline OS Early & 0.0675 & -0.0900 & 0.1161 & -0.0329 \\
\hline OS Late & $-0.3039 * * *$ & -0.1172 & -0.0127 & 0.0005 \\
\hline \multicolumn{5}{|c|}{ Early vs. Late } \\
\hline Early & $-0.7500 *$ & -0.4714 & 0.1485 & - \\
\hline Late & - & - & - & - \\
\hline
\end{tabular}

Table XIII: Correlation between individual preferences and willingness to pay in price list elicitation questions. No individuals in the Information treatment are willing to pay for late resolution, so these correlations do not appear. All subjects in the information treatment report the same measured risk aversion, so this correlation does not appear either.

We also see a strong correlation in the Lottery treatment between a preference for early resolution and risk aversion. Given the risk aversion measure as described in Table XII a higher measured value corresponds to a lower degree of risk aversion. We see a positive correlation between the measured risk aversion parameter and willingness to pay for early resolution. That is, individuals who are less risk averse are more likely to pay for early resolution. This is in line with the false diversification hypothesis-individuals who are more risk averse are more likely to delay uncertainty resolution by diversifying in the second stage of the lottery.

Our results suggest that preferences in the Information treatment are more uniform-individuals prefer early resolution across the board. Results in the Lottery treatment are more determined by individual risk and time preferences. Those who are more patient and less risk averse are more likely to pay for early resolution of uncertainty. However, more research is necessary to confirm these relationships and establish a theoretical link between these preferences.

\section{E. Testing Dillenberger (2010)}

Dillenberger (2010) predicts a preference for one-shot resolution of uncertainty under the following three axioms_-Time Neutrality, Recursivity, and Negative Certainty Independence (NCI). Time neutrality assumes that an individual is indifferent between one-shot early and one-shot late resolution. He does not care when uncertainty resolves as long as it resolves all at once. Recursivity places structure on that way an individual evaluates two-stage lotteries. It states that, in comparing two compound lotteries which differ only in the 
outcome of a single branch, the decision maker compares these two lotteries in the same way that he would compare the two different branches in isolation. NCI is a novel axiom introduced in Dillenberger (2010) which aims to capture common violations of independence in the form of a preference for certainty. NCI states that, if a lottery $p$ is preferred to a sure amount $x$, then preferences should not change when both $p$ and the degenerate lottery $\delta_{x}$ are mixed equivalently with a lottery $q$. That is, if $x$ does not have enough certainty appeal to outweigh lottery $p$ on its own, mixing both with $q$ and thereby eliminating any certainty appeal of $x$ should not result in $\delta_{x}$ being more preferred.

While a rigorous test of the theory is beyond the scope of this paper, we are able to run preliminary tests of all three axioms in our design. The price-list choices between one-shot early and one-shot late resolution allow us to test the time neutrality axiom. Positive willingness to pay for either one-shot early or one-shot late resolution in either treatment implies a violation of time neutrality.

We include one measure of recursivity in our Part 2 elicitation. As part of our measure of risk aversion, we elicit an individual's certainty equivalent, $x$, of the lottery $p=(\$ 6,0.5 ; \$ 0)$. We add a nominal payment to $x, y=x+0.75$, such that the degenerate lottery $\delta_{y}>p$. Recursivity requires that, in evaluating any two compound lotteries that differ only in that one gives $\delta_{y}$ at some branch while the other gives $p$, the lottery giving $\delta_{y}$ should be preferred. We present subjects with the binary choice between two compound lotteries $P=\{p, 0.5 ;(\$ 5,0.75 ; \$ 0), 0.25 ;(\$ 9,0.25 ; \$ 0), 0.25\}$ and $Q=\left\{\$ \delta_{y}, 0.5 ;(\$ 5,0.75 ; \$ 0), 0.25 ;(\$ 9,0.25 ; \$ 0), 0.25\right\}$. These lotteries differ only at a single branch $-P$ gives $p$ with probability 0.5 while $Q$ instead gives $\delta_{y}$ with probability 0.5. A choice of $P$ over $Q$ implies a violation of recursivity.

While a formal test of NCI is beyond the scope of this paper, we include a coarse test of the axiom as part of the elicitation in Part 2. As part of our measure of risk aversion, we elicit an individual's certainty equivalent, $x$, of the lottery $p=(\$ 6,0.5 ; \$ 0)$. Since $p \sim \delta_{x}$, we assume $p>\delta_{x-0.75}$. We mix both $p$ and $\delta_{x-0.75}$ with lottery $q=(0.5, \$ 12 ; \$ 0)$ and offer subjects the binary choice between these two mixtures. NCI requires that $\lambda p+(1-\lambda) q \geq \lambda \delta_{x-0.75}+(1-\lambda) q$. Thus, a choice of $\lambda \delta_{x-0.75}+(1-\lambda) q$ over $\lambda p+(1-\lambda) q$ constitutes a violation of NCI.

As described above, any positive willingness to pay in the time neutrality price list constitutes a violation of the axiom. Table VII reports $40.58 \%$ of subjects violate time neutrality over lotteries, and $50.68 \%$ violate time neutrality over information structures $(p=0.23) .51 .30 \%$ of subjects violate the test of NCI, and $18.26 \%$ violate the test of recursivity. This leaves us with $28.57 \%$ of subjects in the Lottery treatment and only $15.15 \%$ of subjects in the Information treatment who do not violate at least one of the axioms from Dillenberger (2010).

This leaves a small subset on which Dillenberger (2010) makes predictions for one-shot resolution of uncertainty. Even still, we don't find confirmation of the prediction. Of the subjects in the Lottery treatment who satisfy time neutrality, recursivity, and NCI, only $28.57 \%$ choose one-shot resolution in the unrestricted set, 
compared to $25.71 \%$ who do not satisfy all three axioms $(p=0.83)$. Of the $15.15 \%$ in the Information treatment who satisfy the axioms, only $30 \%$ choose one-shot resolution compared to $48.21 \%$ who do not satisfy the axioms $(p=0.29)$. This echos our general finding of the lack of preference for one-shot resolution of uncertainty, even on the smaller subset for which Dillenberger (2010) makes predictions.

\section{F. Internal Consistency}

Given the complexity of our experiment, one may be concerned about within-subject consistency of choices. Our price list questions give evidence that subjects are not indifferent, and qualitative answers to survey questions indicate subjects chose according to well-defined preferences. However, we conduct additional analysis to look at internal consistency of choice.

We consider a subject's choice in the Unrestricted Set to be his true maximum of preference, and measure consistency based on whether the subject chooses this same lottery or information structure whenever available in the restricted sets. Given the very large range of choice possibilities in our experiment, some noise is to be expected. Therefore, we define a subject to be "consistent" if his choice is within some $\epsilon$ of his unrestricted choice. That is, given a choice of $(\bar{p}, \bar{q}, \bar{r})$ in the unrestricted set, a subject is consistent if he chooses $p \in[\bar{p}-\epsilon, \bar{p}+\epsilon], q \in[\bar{q}-\epsilon, \bar{q}+\epsilon]$, and $r \in[\bar{r}-\epsilon, \bar{r}+\epsilon]$, given that $(\bar{p}, \bar{q}, \bar{r})$ was available to choose in the restricted set. We analyze consistency based on $\epsilon=0.05$ and $\epsilon=0.10$.

Table XIV gives the percentage of subjects, per treatment, exhibiting consistency in each set. Consistency is higher in the Information treatment, which is not surprising given the more easily-defined preferences in this environment. Individuals in the Information treatment tend to prefer the earliest resolution, which only leaves one direction for "trembles." Under our less restrictive consistency definition, over 50\% of subjects are consistent in each Information set, while around $40 \%$ are consistent in the Lottery sets.

Inconsistency could be due to a number of factors. First, these could be indifferent subjects who are choosing randomly. However, this doesn't seem to be the case. In the Information treatment, there is no significant difference in consistency among those who express positive willingness to pay and those who don't (42\% vs. $53 \%, p=0.28$ for $\epsilon=0.05$ and $52 \%$ vs. $60 \%, p=0.41$ for the $\epsilon=0.10$ definition of consistency). In the lottery treatment, subjects who express positive willingness to pay are actually less consistent (18\% vs. $42 \%$, $p=0.0075$ and $36 \%$ vs. $51 \%, p=0.10$ ). Second, it could be an expression of stochastic choice or a preference for randomization across lottery sets. Finally, it could be simply mistakes in subjects' choices. Internal consistency across lotteries is not the main focus of our paper, but it leaves interesting questions for future research. 


\begin{tabular}{lcccc}
\hline & \multicolumn{2}{c}{ 0.05-Unit Square } & \multicolumn{2}{c}{ 0.10-Unit Square } \\
& Lotteries & Information & Lotteries & Information \\
\hline Set 2 & $30.43 \%$ & $48.33 \%$ & $43.48 \%$ & $56.67 \%$ \\
Set 3 & $29.55 \%$ & $52.46 \%$ & $45.45 \%$ & $60.66 \%$ \\
Set 4 & $22.22 \%$ & $33.33 \%$ & $47.22 \%$ & $50.00 \%$ \\
Set 5 & $25.00 \%$ & $33.33 \%$ & $37.50 \%$ & $50.00 \%$ \\
Set 6 & $29.27 \%$ & $25.00 \%$ & $36.59 \%$ & $50.00 \%$ \\
Set 7 & $28.00 \%$ & $52.46 \%$ & $32.00 \%$ & $62.30 \%$ \\
Set 8 & $21.88 \%$ & $52.38 \%$ & $37.50 \%$ & $60.32 \%$ \\
Set 9 & $23.33 \%$ & $43.94 \%$ & $40.00 \%$ & $50.00 \%$ \\
\hline
\end{tabular}

Table XIV: Percentage of subjects who choose their unrestricted set choice conditional on it being available

Internal consistency varies by subset in the population. Looking at choices in the unrestricted set, we classify a subjects as making choices in line with the predictions of a model if he chooses the earliest, latest, suspense-optimal, or surprise-optimal choice. The remaining subjects, $62 \%$ in the Information treatment and $41 \%$ in the Lottery treatment, choose gradually resolving uncertainty which is not specifically predicted by any model. Under our $\epsilon=0.10$ definition of consistency, we find that, in the Lottery treatment, subjects who make choices in line with a model are consistent $51 \%$ of the time while those who choose other gradually resolving uncertainty are consistent $34 \%$ of the time $(p=0.047)$. In the Information treatment, subjects choosing in line with a model are consistent $68 \%$ of the time, while those choosing other gradually resolving lotteries are consistent only $36 \%$ of the time $(p=0.0002)$.

\section{G. InCEntive Compatibility}

Subjects made choices in 9 different Lottery sets, and additionally answered questions for 3 different price list scenarios, each of which contained 21 questions. We randomly selected one of these for payment ${ }^{30}$ Azrieli et al. (forthcoming) show that this random problem selection mechanism elicits true preferences in each question under a monotonicity assumption. However, the process of randomly selecting a scenario to implement creates an additional stage of the lottery, a "stage 0" before the chosen two-stage lottery plays out. A subject who prefers one-shot resolution has no option to resolve all uncertainty in one of the stages of this "three-stage" lottery. If subjects view the random problem selection mechanism as part of the lottery, this could critically influence the exact preferences we are trying to study.

We run a control treatment with 40 subjects, where subjects choose their most preferred lottery from only the unrestricted set. Here, a subject with a strict preference for one-shot resolution can guarantee this by choosing one-shot early or late in the single choice set. We compare the proportion of one-shot choices in the

\footnotetext{
${ }^{30}$ To determine which scenario played out, the computer uniformly drew a random number between 1 and 12 , where 1-9 corresponded to the Lottery Sets and 10-12 corresponded to the price list scenarios. If the computer drew 10-12, it would then draw a number 1-21 to determine which row of the price list to implement.
} 
control treatment to those in the main experiment. If subjects in the main experiment integrate the payment mechanism into the lottery and view it as a three-stage lottery, therefore unable to resolve all uncertainty in one stage, we would expect a lower proportion of one-shot choices than in the control treatment where they can resolve uncertainty in a single stage.

We find no difference in frequency of one-shot choices between the control and main treatments. In the Lottery treatment, $30.43 \%$ of subjects choose one-shot resolution in the main experiment while $33.3 \%$ choose one-shot resolution in the control treatment $(p=0.814)$. In the information treatment, $49.32 \%$ choose one-shot resolution in the main treatment while $54.55 \%$ choose one-shot resolution in the control treatment $(p=0.669)$. We conclude that it does not appear subjects integrate the random problem selection lottery into the main lottery of the experiment. As a result, it does not interact with a preference for one-shot resolution.

\section{H. Venmo Payments}

Andreoni and Sprenger have recently addressed numerous concerns surrounding payments from incentivized intertemporal choice questions (Andreoni and Sprenger, 2012a b). In particular, choices between payments "now" and payments in the future require transaction costs to be exactly equal at both time periods. That is, subjects should not prefer payments "now" because they are more convenient, in cash compared to checks, etc.

We address this by paying all Part 2 payments through Venmo, a money transfer app. In the recruitment email, subjects were told that there is a possibility of being paid through Venmo and they must already have an account set up. Part 2 instructions indicate that a random question would be selected for payment and money would be directly transferred to them through Venmo according to their choice on that question. Both "now" and "later" payments were paid through Venmo, and subjects would receive Venmo payment regardless of whether a risk or time elicitation question were randomly selected. 


\section{Lottery Treatment}

\section{Instructions: Part 1}

Welcome to our decision making study! Thank you for your participation. Please turn off and put away your cell phones, put away any books or other things you've brought with you, and please refrain from talking to other participants during the study.

Here in the Economics Experimental Lab, our research does not involve deception of any kind. You might have participated in experiments elsewhere on campus where that was not the case, but it will always be true here. What this means is that, in this Lab, we will provide you with all relevant information and we will be truthful. The instructions accurately reflect how decisions and processes will unfold. We will not deceive or lie to you in any way.

This study will take approximately 90 minutes. You will receive $\$ 10$ for showing up on time. In addition to this "show up payment," you may earn additional money throughout the study. Your earnings may depend on your decisions and on elements of chance.

There are two separate "Parts" to this experiment, and each Part has multiple Tasks. These instructions are for Part 1. Part 2 will take place after Part 1 is finished. Your decisions in Part 1 will not in any way affect your decisions or payoffs in Part 2.

\section{Task 1: Lotteries}

\section{The Lottery}

Your earnings from Part 1 will be determined by the outcome of a lottery which will take place in two steps. We will represent the lottery as drawing a ball from an urn.

- There are 2 possible urns the ball could be drawn from, Urn 1 and Urn 2.

- Each urn will have some balls in it, and the balls can be either red or blue.

- In Step 1 of the lottery, we will pick either Urn 1 or Urn 2.

- In Step 2 of the lottery, we will draw a ball from the selected urn.

- Step 2 will not occur immediately after Step 1 - some time will pass in between (more on this below)

\section{Payoffs}

The color of the chosen ball will determine your payoff from the lottery.

- If the ball drawn from the urn is red, you will receive the high prize -- $\$ 11$.

- If the ball drawn from the urn is blue, you will receive the low prize -- \$2. 


\section{Overall Odds}

Overall, there is a $50 \%$ chance that a red ball will be drawn and a $50 \%$ chance that a blue ball will be drawn. This means that there's a $50 \%$ chance you will earn the high prize and a $50 \%$ chance you will earn the low prize.

\section{Your Choice}

There are many different possible lotteries that can be played, all with the same Overall Odds. You will be choosing the lottery that you want to participate in. All the available lotteries have the same Overall Odds and same Payoffs. Essentially, you will decide how many balls of each color you would like in Urn 1 and Urn 2, and you will determine the probability of choosing Urn 1 and Urn 2 in Step 1 of the lottery.

\section{Choosing Lotteries}

Every lottery will have the same Overall Odds, $50 \%$ chance of drawing a red ball and $50 \%$ chance of drawing a blue ball. However, there are many possible lotteries that have the same Overall Odds. There are three things that determine a lottery, and you will be choosing these three things -

1. The fraction of red and blue balls in Urn 1

2. The fraction of red and blue balls in Urn 2

3. The likelihood of selecting Urn 1 vs. Urn 2

When you make your decisions, your screen will have three "sliders" on it. The slider on top determines the likelihood of choosing Urn 1 vs. Urn 2. The bottom left slider determines the fraction of red and blue balls in Urn 1, and the bottom right slider determines the fraction of red and blue balls in Urn 2 .

On your screen, you'll also see three "Auto" buttons, one corresponding to each slider. You must put exactly one slider on "Auto" at all times. You can put any of the three sliders on Auto, and then you can adjust the other two in any way you want. This way, the computer will automatically adjust the Auto slider in order to maintain the Overall Odds. We'll go through some examples in a minute to give you an idea how the computer interface works.

\section{Lottery Details}

The first important thing to realize is that the Overall Odds of drawing a red ball or a blue ball does not change when you make your decisions or adjust the sliders. No matter which lottery you choose, overall there is a $50 \%$ chance of drawing a red ball and a $50 \%$ chance of drawing a blue ball.

- When you choose your most preferred lottery, you are choosing how you want the odds to be determined in Step 1 relative to Step 2.

- For example, in Lottery 4 in the table, the outcome is ultimately determined in Step 1, since after the Urn is selected the outcome is known. You will either know for sure that we will draw a red ball and you will earn $\$ 11$, or you will know for sure that we will draw a blue ball and you will earn \$2.

- On the other hand, in Lottery 1 above, you will know after drawing Urn 1 or Urn 2 that you still have a $50 \%$ chance of drawing a red ball and a $50 \%$ chance of drawing a blue ball. 
- For Lotteries 2 and 3, the outcome is determined partially in Step 1 and partially in Step 2, since the chosen Urn will have a higher or lower fraction of red (blue) balls than the Overall Odds, but there is still a chance of drawing a red (blue) ball from either Urn.

- You are choosing how to spread the odds across Step 1 and Step 2, which is also like choosing what you want Step 1 to tell you about your chances of drawing a red (blue) ball later.

Another important thing to realize is that it can't be the case where both Urns have more than $50 \%$ red balls and it also can't be the case where both Urns have fewer than $50 \%$ red balls.

- It has to be that one Urn has more than or equal to $50 \%$ red balls, but the other Urn has less than or equal to $50 \%$ red balls. This way, the Urns will "average" out to equal a $50 \%$ chance of drawing a red ball.

- The computer will only allow you to choose options that satisfy these conditions. Urn 1 will have fewer than $50 \%$ red balls and Urn 2 will have more than $50 \%$ red balls.

\section{Restrictions}

You'll be choosing your most preferred lottery under various conditions which we will call "scenarios." Remember, your most preferred lottery is determined by (i) The fraction of red and blue balls in Urn 1, (ii) The fraction of red and blue balls in Urn 2, and (iii) The likelihood of selecting Urn 1 vs. Urn 2.

- In some scenarios, the computer might place "restrictions" on one or more of these things.

- For example, the computer might require that Urn 1 contains at least $10 \%$ red balls, or that Urn 2 contains no more than $80 \%$ red balls.

\section{Preferences}

There are no right or wrong answers in any of these scenarios. We are simply interested in your preferences, so please consider the options carefully and choose the one lottery you most prefer in each scenario. In fact, you should answer each question as if it will directly determine your Part 1 earnings, since one of the scenarios will. If you don't answer according to your actual preferences, you might end up with something you prefer less than another available option.

\section{Experiment Timing}

\section{Task 1}

Part a:

You will have the chance to participate in a practice lottery, so you can get used to how the computer sliders work, how to pick your preferred lotteries, etc. As you're getting familiar with the task, we will also ask you a few comprehension questions.

Part b:

Next, you'll participate in the Lottery Task described above. You will choose your most preferred lottery from 12 different scenarios. These scenarios might differ in the restrictions that the computer places on the Urns. 
Part c:

After you make all your decisions, we will determine which of the 12 scenarios will actually play out. We'll do this in the following way. The computer will randomly draw a number 1-12. Each number is equally likely to be drawn. The number chosen will correspond to the scenario that will play out. You will participate in whatever lottery you chose as your preferred lottery in that scenario.

Part d:

Now that the lottery has been selected, we will determine whether the ball will be drawn from Urn 1 or Urn 2 according to the probabilities you have chosen in your preferred lottery.

How will we do that? Here is an example. Let's say, in your most preferred lottery, Urn 1 will be chosen with $35 \%$ chance and Urn 2 will be chosen with $65 \%$ chance.

- The computer will randomly draw a number 1-100. Each number is equally likely to be drawn.

- If the number drawn is less than or equal to 35, Urn 1 will be chosen.

- If the number drawn is greater than 35, Urn 2 will be chosen.

Task 2 and 3

After we select the Urn, you will participate in Tasks 2 and 3 of the experiment. This means that, after Part $d$, you will be told which Urn has been chosen and you will be reminded the proportion of red and blue in that Urn. But we will not randomly draw a ball from the Urn until the very end of Part 1 . So when you participate in Tasks 2 and 3, you will know the Urn that will eventually determine your payoffs from Part 1, but we will not draw a ball to determine the lottery outcome until the end of Tasks 2 and 3 . Tasks 2 and 3 will take about 30 minutes, so you will be waiting for those 30 minutes to learn the outcome of the lottery.

\section{Task 2: Coloring}

After you have been notified which Urn the ball will be drawn from, you'll participate in Task 2. In Task 2 , you will use the computer to "color" a representation of your chosen Urn.

More detailed instructions on the coloring task will follow.

\section{Task 3: Questions}

We will also ask you various other questions about lotteries and your choices. These questions will be hypothetical, to test your understanding of the lotteries and ask you questions about your preferences.

Remember, the actual Urn determining your payoffs will have already been chosen, but we will not have selected a ball yet to determine your payoffs. 


\section{Lottery Outcome}

After Task 3, we will determine the actual outcome of the lottery. We will randomly choose a number 1100. You've colored the 100 balls in your urn in Task 2, so the random number drawn corresponds to one of the colored balls.

If the number drawn corresponds to a ball you've colored red, you will earn the high prize of \$11. If it's blue, you will earn the low prize of $\$ 2$.

\section{Part 2}

After the outcome is revealed and Part 1 is finished, you will receive the instructions for Part 2, which is a short additional "bonus" task. Your decisions in Part 1 will not in any way affect your decisions or payoffs in Part 2. The two parts are completely independent. 


\section{Information Treatment}

\section{Instructions: Part 1}

Welcome to our decision making study! Thank you for your participation. Please turn off and put away your cell phones, put away any books or other things you've brought with you, and please refrain from talking to other participants during the study.

Here in the Economics Experimental Lab, our research does not involve deception of any kind. You might have participated in experiments elsewhere on campus where that was not the case, but it will always be true here. What this means is that, in this Lab, we will provide you with all relevant information and we will be truthful. The instructions accurately reflect how decisions and processes will unfold. We will not deceive or lie to you in any way.

This study will take approximately 90 minutes. You will receive $\$ 10$ for showing up on time. In addition to this "show up payment," you may earn additional money throughout the study. Your earnings may depend on your decisions and on elements of chance.

There are two separate "Parts" to this experiment, and each Part has multiple Tasks. These instructions are for Part 1. Part 2 will take place after Part 1 is finished. Your decisions in Part 1 will not in any way affect your decisions or payoffs in Part 2.

\section{Task 1: Signals}

\section{The Lottery}

Your earnings from Part 1 will be determined by the outcome of a lottery. At the beginning of the experiment, the computer will draw a random number between 1 and 100. Each number is equally likely to be drawn. If the computer draws 1-50, you will win the "low prize." If the computer draws 51-100, you will win the "high prize." This means there's a 50\% chance you've won the high prize and a $50 \%$ chance you've won the low prize.

You will receive information about the outcome of the lottery in two steps. The computer won't reveal the number drawn, so you won't learn the outcome of the lottery immediately. However, you can receive some earlier information about the outcome, which we'll call a "signal." So the first step in learning the outcome of the lottery is the information in the signal you will receive. In the second step, you'll learn the final outcome.

\section{Payoffs}

The randomly selected number will determine your payoff from the lottery.

- If you win the high prize, you'll earn $\$ 11$.

- If you win the low prize, you'll earn $\$ 2$.

\section{Overall Odds}


Overall, there is a $50 \%$ chance of winning the high prize and a $50 \%$ chance of winning the low prize. Note that the chance of winning and losing is equal for everyone. This means there's a $50 \%$ chance you'll earn $\$ 11$ from the lottery and a 50\% chance you'll earn \$2.

\section{Your Choice}

There are many possible signals that you could receive to get information about the outcome of the lottery. You will be choosing the signal that you want to receive. The type of signal that you receive does not change the Overall Odds or the likelihood of receiving the high prize or the low prize. You will choose the type of information you want to receive about the outcome of the lottery.

\section{Choosing Signals}

Though you will not learn the lottery outcome immediately, the computer will know whether you've won the high prize or the low prize. As a result, the computer can give you a "signal" about the outcome. The signal is just a piece of information that might tell you more about whether you've won the high or low prize. You will choose the kind of signal you want.

The amount and type of information varies across these signals. You will pick your most preferred information option from all of these possibilities. So you will get to determine the type of information you'd like the signal to tell you about the outcome of the lottery.

You'll do that in the following way. There are two "Urns," Urn 1 and Urn 2. You can visualize them as being filled with red and/or blue balls. The computer will randomly pick one ball from one of the Urns; this selected ball is the "signal" you will see. The computer will show you a ball from Urn 1 if you've won the low prize, and it will show you a ball from Urn 2 if you've won the high prize. You won't learn which Urn the ball was drawn from; you'll just see the ball color.

Each possible signal you get is determined by three things:

1. The likelihood of seeing a red or blue signal

2. The chance that you've won the high prize, given that you see a blue signal

3. The chance that you've won the high prize, given that you see a red signal

You'll be choosing these three things. When you make your decisions, your screen will have three "sliders" on it. The slider on top determines the likelihood of seeing a red or blue signal. The bottom left slider determines the chance that you've won the high prize, given that you see a blue signal, and the bottom right slider determines the chance that you've won the high prize, given that you see a red signal.

On your screen, you'll also see three "Auto" buttons, one corresponding to each slider. You must put exactly one slider on "Auto" at all times. You can put any of the three sliders on Auto, and then you can adjust the other two in any way you want. This way, the computer will automatically adjust the Auto slider in order to maintain the Overall Odds. We'll go through some examples in a minute to give you an idea how the computer interface works.

\section{Lottery Details}

- The first important thing to realize is that the likelihood of winning the high prize or low prize does not change when you make your decisions or adjust the sliders. No matter what type of 
information you choose, there is a $50 \%$ chance of winning the high prize and a $50 \%$ chance of winning the low prize. No information choices affect the number that the computer draws to determine whether you win the high or low prize.

- When you choose your information, you are choosing what you want to learn from the signal you see.

- For example, in Information 4 in the table shown, the signal tells you for sure whether you've won the high or low prize.

- On the other hand, in Information 1 shown, the signal does not give you any information about the lottery outcome. You will know that there is a 50\% chance you've won the high prize and a $50 \%$ chance you've won the low prize, regardless of the signal you see.

- For Lotteries 2 and 3, the signals give you some, but not all, information about whether you've won the high or low prize.

Another important thing to note is that the computer is set up so that there will always be a less than or equal to $50 \%$ chance you've won the high prize if you see a blue ball and a greater than or equal to $50 \%$ chance you've won the high prize if you see a red ball.

- This ensures that, if you see a red ball as your signal, it's more likely to have come from Urn 2, which means it's more likely that you've won the high prize.

- Thus, seeing a red ball means that your chances of having won the high prize are either equal to or higher than $50 \%$, and seeing a blue ball means that your chances are either equal to or lower than $50 \%$.

- How much your chances of having won changes after you see a red or blue ball depends on the contents of the Urns.

\section{Restrictions}

You'll be choosing your Information under various conditions which we will call "scenarios." Remember, your information is determined by (i) The likelihood of seeing a red or blue signal, (ii) The chance that you've won the high prize, given that you see a blue signal, and (iii) The chance that you've won the high prize, given that you see a red signal.

- In some scenarios, the computer might place "restrictions" on one or more of these things.

- For example, the computer might require that there's no more than a $20 \%$ chance you've won the high prize, given that you see a blue signal.

\section{Preferences}

There are no right or wrong answers in any of these scenarios. We are simply interested in your preferences, so please consider the options carefully and choose the one lottery you most prefer in each scenario. In fact, you should answer each question as if it will directly determine your Part 1 earnings, since one of the scenarios will. If you don't answer according to your actual preferences, you might end up with something you prefer less than another available option. 


\section{Experiment Timing}

Task 1

Part a:

You will have the chance to participate in a practice scenario, so you can get used to how the computer sliders work, how to pick your preferred signals, etc. As you're getting familiar with the task, we will also ask you a few comprehension questions.

Part b:

Next, you'll participate in the Task described above. You will choose your information from 9 different scenarios. These scenarios might differ in the restrictions that the computer places on the Urns.

Part c:

After you make all your decisions, we will determine which of the 9 scenarios will actually play out. We'll do this in the following way. The computer will randomly draw a number 1-9. Each number is equally likely to be drawn. The number chosen will correspond to the scenario that will play out. You will receive a signal according to how you chose in that scenario.

Part d:

Now that the information has been selected, you will see a signal according to the information you have chosen in that scenario. Here's how that works.

Let's say you've decided to make Urn $130 \%$ red balls and make Urn 2 80\% red balls. If you've won the high prize, the computer will draw a ball from Urn 2, in which case there's an $80 \%$ chance you'll see a red ball as your signal. If you've won the low prize, the computer will draw a ball from Urn 1, in which case there's a $30 \%$ chance you'll see a red ball.

You will only see the signal, but the computer will tell you how likely it is that you've won the high prize or the low prize, given the signal you see.

\section{Task 2 and 3}

After you see the signal, you will participate in Tasks 2 and 3 of the experiment. This means that, after Part $d$, you will know the information from the signal, but we will not tell you whether you've won the high or low prize until the very end of Part 1. So when you participate in Tasks 2 and 3, you will know the information from the signal, but you won't learn anything else about the lottery outcome until the end of Tasks 2 and 3. Tasks 2 and 3 will take about 30 minutes, so you'll be waiting for those 30 minutes to learn the outcome of the lottery. 


\section{Task 2: Coloring}

After you see your signal, you'll participate in Task 2. In Task 2, you will use the computer to "color" a representation of how likely it is that you've won the high prize or the low prize.

On your computer, you will see 100 circles labeled 1-100. You will color them red or blue according to the information from your signal. For example, imagine that you see a blue signal, and according to the information you've chosen, this means that there's a 30\% chance you've won the high prize and a 70\% chance you've won the low prize. In task 2, you would color balls 1-30 red and 31-100 blue. The computer will not allow you to color the balls in any other way. This is just to create a visual for yourself of your chances of winning the prizes.

More detailed instructions on the coloring task will follow.

\section{Task 3: Questions}

Task 3 will present you with various possible information specifications and we will ask you to answer various questions. These questions will be hypothetical. Please read the questions carefully before answering, and raise your hand with any questions.

Remember, it will have already been decided whether you've won the high prize or the low prize, and you will have already seen your signal. However, we will tell you the lottery outcome at the very end of Task 1.

\section{Lottery Outcome}

After Task 3, we will tell you the actual outcome of the lottery. The computer will reveal whether you won the high or low prize.

\section{Part 2}

After the outcome is revealed and Part 1 is finished, you will receive the instructions for Part 2, which is a short additional "bonus" task. Your decisions in Part 1 will not in any way affect your decisions or payoffs in Part 2. The two parts are completely independent. 


\section{Instructions: Part 2}

In this final Part, you will be asked to answer a number of questions. The questions all take a similar format, but each is unique. At the end of the experiment, one question will be selected at random from all of the questions you answered. The amount of money you will earn from this Task will depend on your answers to this question.

Any earnings you receive in this part will be paid to you directly through Venmo.

Most questions will take the form of lists of choices. There will be multiple choices within one "Decision Stage." For example, Decision Stage 1 might ask you to choose between receiving some amount of money (say \$6) in one week's time, or different amounts of money now. In such a case, Question 1 would look like this:

\begin{tabular}{|c|c|}
\hline$\$ 6$ in 1 week & $\$ 0.50$ today \\
\hline$\$ 6$ in 1 week & $\$ 1$ today \\
\hline$\$ 6$ in 1 week & $\$ 1.50$ today \\
\hline$\$ 6$ in 1 week & $\$ 2$ today \\
\hline$\$ 6$ in 1 week & $\$ 2.50$ today \\
\hline$\$ 6$ in 1 week & $\$ 3$ today \\
\hline$\$ 6$ in 1 week & $\$ 3.50$ today \\
\hline$\$ 6$ in 1 week & $\$ 4$ today \\
\hline$\$ 6$ in 1 week & $\$ 4.50$ today \\
\hline$\$ 6$ in 1 week & $\$ 5$ today \\
\hline$\$ 6$ in 1 week & $\$ 5.50$ today \\
\hline$\$ 6$ in 1 week & $\$ 6$ today \\
\hline$\$ 6$ in 1 week & $\$ 6.50$ today \\
\hline
\end{tabular}

This Decision Stage is actually asking you 13 different questions, one for each row. For each row, you must choose between the option on the left or the option on the right. Note that on each line, the option on the left stays the same in each row while the option on the right increases as you go down the list.

In each row, you select the option you like by clicking the box next to that option.

If Decision Stage 1 were selected as the one that will be paid at the end of the experiment, ONE row will be selected at random from those in Decision Stage 1 and you will be paid according to 
your choice on that row. That is, if Decision Stage 1 were selected, a row would be randomly chosen between the first row ( $\$ 6$ in 1 week vs. $\$ 0$ today) and the last row (\$6 in 1 week vs. $\$ 6$ today) with equal probability. Let's say, for example, the second row were chosen. Then your payment for this Task of the experiment would depend on your choice in the second row. If you had chosen "\$6 in 1 week", then that is what you would receive -- \$6 transferred to you through VenMo, one week from today. If you had chosen " $\$ 0.50$ today", then that is what you would receive -- $\$ 0.50$ transferred to your VenMo account today, immediately after the experiment.

At the start, all boxes will be unchecked. You must check exactly one box in each row. You can change your answer anytime before submitting. Most people start out preferring Option A and then may or may not switch over to preferring Option B. If you switch to Option B, you should not switch back to preferring Option A in later rows.

There are no right or wrong answers to any of these questions. We are simply interested in your preferences, so please consider the options carefully and answer according to what you prefer. In fact, you should answer each question as if it will directly determine your Part 2 earnings, since one of the rows will. If you don't answer according to your actual preferences, you might end up with something you prefer less than the other available option.

There are a few different types of these questions that we will ask, so we will go through them now in a little more detail.

\section{Future Questions:}

In this section, you will be asked questions about amounts of money that you may receive in the future. In particular, these questions will concern amounts of money that you may receive after some time delay -- for example, it might be that you receive $\$ 10$ in 5 weeks.

The amount of money will always be transferred through VenMo directly to you at the date specified. So in the example above, if you were to receive $\$ 10$ in 5 weeks, we would directly transfer $\$ 10$ into your VenMo account, 5 weeks from today.

All payments are guaranteed to arrive, certified by the Ohio State Experimental Economics Laboratory. If the question selected involves future payments, I will schedule the payment transfer before you leave the lab today. This way, you can ensure your payment will arrive exactly when I say it will. You have received a slip of paper with my personal phone number and email address on it. Keep this. If, for some reason, you do not receive your payment at the scheduled time, you can call/text/email me and receive payment immediately. 


\section{Lottery Questions:}

For this questions, you will be asked questions about amounts of money that might be determined by a "lottery." All this means is that there is some chance you will receive a given amount. For example, it might be that you have a $75 \%$ chance of receiving $\$ 10$ and a $25 \%$ chance of receiving $\$ 2$.

If one of these questions is chosen for payment, you will receive the amount determined by the lottery. You will receive the payment today through VenMo.

To help understand the lotteries, here's an example. Imagine a lottery that gives you a $75 \%$ chance of receiving $\$ 10$ and a $25 \%$ chance of receiving $\$ 2$. The computer will draw a random number to determine the lottery outcome. You can think of this in the following way. Imagine there are 100 balls, numbered 1-100, and we're going to randomly draw one of them to determine the lottery outcome. A $75 \%$ chance of receiving $\$ 10$ means that if we draw ball number $1-75$, you would earn $\$ 10$. If we draw $76-100$, you would earn $\$ 2$. Since there are 75 out of 100 balls numbered $1-75$, this is exactly a $75 \%$ chance of earning $\$ 10$. There are 25 balls numbered $76-100$, so this is a $25 \%$ chance of earning $\$ 2$.

Here is an example of what questions in this section could look like. Remember, you are making a choice for EACH row, and one of the rows could be selected and you will be paid based on your answer in that row:

\begin{tabular}{|c|c|}
\hline $75 \%$ chance of $\$ 10,25 \%$ chance of $\$ 2$ & $100 \%$ chance of $\$ 5$ \\
\hline $75 \%$ chance of $\$ 10,25 \%$ chance of $\$ 2$ & $100 \%$ chance of $\$ 5.50$ \\
\hline $75 \%$ chance of $\$ 10,25 \%$ chance of $\$ 2$ & $100 \%$ chance of $\$ 6$ \\
\hline $75 \%$ chance of $\$ 10,25 \%$ chance of $\$ 2$ & $100 \%$ chance of $\$ 7$ \\
\hline $75 \%$ chance of $\$ 10,25 \%$ chance of $\$ 2$ & $100 \%$ chance of $\$ 7.50$ \\
\hline $75 \%$ chance of $\$ 10,25 \%$ chance of $\$ 2$ & $100 \%$ chance of $\$ 8$ \\
\hline $75 \%$ chance of $\$ 10,25 \%$ chance of $\$ 2$ & $100 \%$ chance of $\$ 8.50$ \\
\hline $75 \%$ chance of $\$ 10,25 \%$ chance of $\$ 2$ & $100 \%$ chance of $\$ 9$ \\
\hline $75 \%$ chance of $\$ 10,25 \%$ chance of $\$ 2$ & $100 \%$ chance of $\$ 9.50$ \\
\hline $75 \%$ chance of $\$ 10,25 \%$ chance of $\$ 2$ & $100 \%$ chance of $\$ 10$ \\
\hline $75 \%$ chance of $\$ 10,25 \%$ chance of $\$ 2$ & $100 \%$ chance of $\$ 10.50$ \\
\hline $75 \%$ chance of $\$ 10,25 \%$ chance of $\$ 2$ & $100 \%$ chance of $\$ 11$ \\
\hline $75 \%$ chance of $\$ 10,25 \%$ chance of $\$ 2$ & \\
\hline
\end{tabular}




\section{Multi-Stage Lottery Questions:}

For these questions, you will be asked questions about amounts of money that might be determined by a "lottery." These are similar to the Lottery Questions we just discussed, but the lotteries might have multiple stages. For example, it might be that there is a $50 \%$ chance of playing Lottery 1 and a $50 \%$ chance of playing Lottery 2, where Lottery 1 gives you a $50 \%$ chance of winning $\$ 10$ and a $50 \%$ chance of winning $\$ 2$ while Lottery 2 gives you a $75 \%$ chance of winning $\$ 10$ and a $25 \%$ chance of winning $\$ 2$.

In this example given above, the computer would first draw a random number 1-100. If the number is 1-50, you would play Lottery 1. If it's 51-100, you would play Lottery 2.

Let's imagine the computer drew number 74 , so you're playing Lottery 2 . Lottery 2 gives you a $75 \%$ chance of winning $\$ 10$ and a $25 \%$ chance of winning $\$ 2$. So the computer would draw another random number, totally independent from the first number. This is just any number 1100. If the number is $1-75$, you would win $\$ 10$. If it's $76-100$, you would win $\$ 2$.

This type of question would look like this:

\begin{tabular}{|c|c|}
\hline $50 \%$ chance Lottery $1,50 \%$ chance Lottery 2 & $100 \%$ chance of $\$ 5$ \\
\hline $50 \%$ chance Lottery $1,50 \%$ chance Lottery 2 & $100 \%$ chance of $\$ 5.50$ \\
\hline $50 \%$ chance Lottery $1,50 \%$ chance Lottery 2 & $100 \%$ chance of $\$ 6$ \\
\hline $50 \%$ chance Lottery $1,50 \%$ chance Lottery 2 & $100 \%$ chance of $\$ 6.50$ \\
\hline $50 \%$ chance Lottery $1,50 \%$ chance Lottery 2 & $100 \%$ chance of $\$ 7$ \\
\hline $50 \%$ chance Lottery $1,50 \%$ chance Lottery 2 & $100 \%$ chance of $\$ 7.50$ \\
\hline $50 \%$ chance Lottery $1,50 \%$ chance Lottery 2 & $100 \%$ chance of $\$ 8$ \\
\hline $50 \%$ chance Lottery $1,50 \%$ chance Lottery 2 & $100 \%$ chance of $\$ 8.50$ \\
\hline $50 \%$ chance Lottery $1,50 \%$ chance Lottery 2 & $100 \%$ chance of $\$ 9$ \\
\hline $50 \%$ chance Lottery $1,50 \%$ chance Lottery 2 & $100 \%$ chance of $\$ 9.50$ \\
\hline $50 \%$ chance Lottery $1,50 \%$ chance Lottery 2 & $100 \%$ chance of $\$ 10$ \\
\hline $50 \%$ chance Lottery $1,50 \%$ chance Lottery 2 & $100 \%$ chance of $\$ 10.50$ \\
\hline $50 \%$ chance Lottery $1,50 \%$ chance Lottery 2 & $100 \%$ chance of $\$ 11$ \\
\hline
\end{tabular}

Again, there are no right or wrong answers to any of these questions. We are simply interested in studying your preferences, so please take your time to consider the options and choose which you prefer. 
Lottery 1:

Overall Odds:

(50\% red, $50 \%$ blue )
Urn 1 will be chosen with probability

Urn 2 will be chosen with probability 37

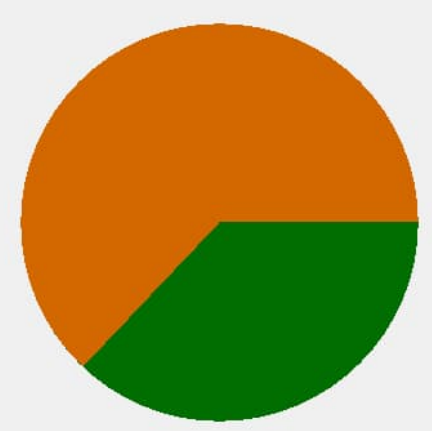

AUTO 100

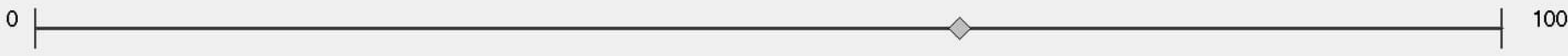

Urn 1 AUTO

50

0

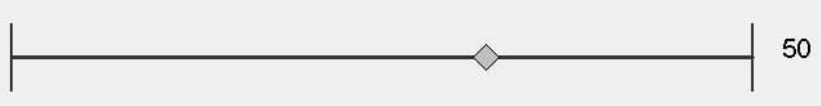

Urn 2

50

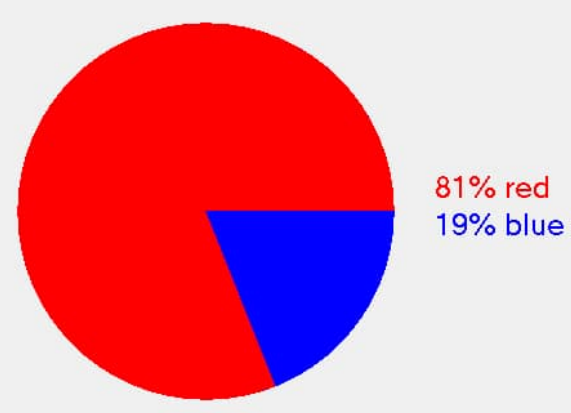

100 


\section{Urn 1}

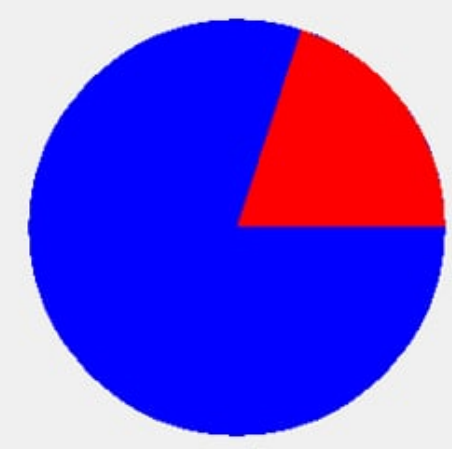

$20 \%$ red

$80 \%$ blue

The balls numbered less than or equal to 20 are RED, and the balls colored greater than 20 are colored BLUE .

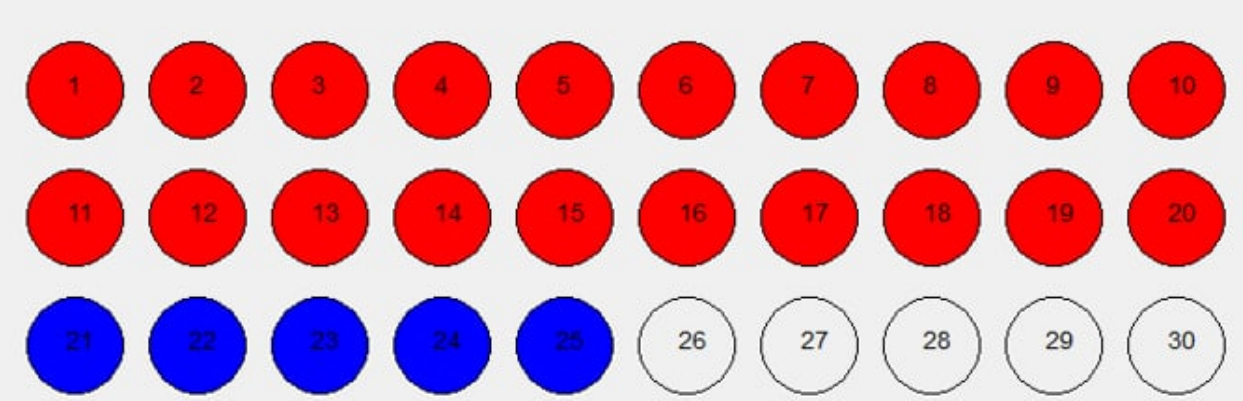

(31) 32 (33 34 35 37 39 30

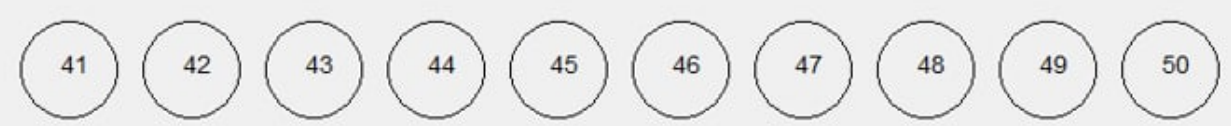

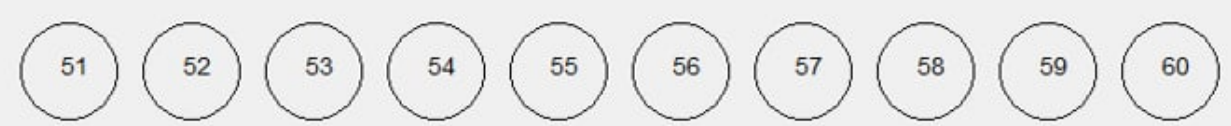

(61) $62 \rightarrow 63 \rightarrow 65 \rightarrow 68 \rightarrow 70$

(71) $72 \rightarrow 73(74) 76>79>70$

(81) $82,83 \rightarrow 85>89>80$

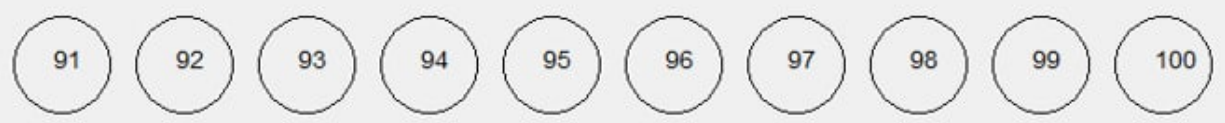

